

\section{Current progress in real-time functional magnetic resonance-based neurofeedback: methodological challenges and achievements}

Christian Paret*1,2 Noam Goldway2, 3 Catharina Zich4,5 Jackob Nimrod Keynan 2,7 Talma Hendler2,3, 7 David Linden 8,9 Kathrin Cohen Kadosh*4,6

1 Department of Psychosomatic Medicine and Psychotherapy, Central Institute of Mental Health Mannheim, Medical Faculty Mannheim / Heidelberg University, Germany

2 Sagol Brain Institute, Wohl Institute for Advanced Imaging, Tel-Aviv Sourasky Medical Centre, TelAviv, Israel

3 Sagol School of Neuroscience Tel Aviv University

4 Department of Experimental Psychology, University of Oxford, Oxford, OX1 3UD, UK

5 Department of Psychology, University of Oldenburg, 26111 Oldenburg, Germany

6 School of Psychology, University of Surrey, Guildford GU2 7XH, UK

7 School of Psychological Science and Faculty of Medicine, Tel Aviv University

8 MRC Centre for Neuropsychiatric Genetics and Genomics, Division of Psychological Medicine and Clinical Neurosciences, School of Medicine, Cardiff University, Cardiff CF24 4HQ, United Kingdom

9 School of Mental Health and Neuroscience, Faculty of Health, Medicine and Life Sciences, Maastricht University, Universiteitssingel 40, 6229 ER Maastricht, The Netherlands

* Corresponding authors:

Kathrin Cohen Kadosh, kathrin.cohenkadosh@psy.ox.ac.uk

School of Psychology, University of Surrey, Guildford, Surrey GU2 7XH, UK

Christian Paret, christian.paret@zi-mannheim.de,

Central Institute of Mental Health, J5, D-68159 Mannheim, Germany

\section{Abstract}

Neurofeedback (NF) is a research and clinical technique, characterized by live demonstration of brain activation to the subject. The technique has become increasingly popular as a tool for the training of brain self-regulation, fueled by the superiority in spatial resolution and fidelity brought along with real-time analysis of $\mathrm{fMRI}$ (functional magnetic resonance imaging) data, compared to the more traditional EEG (electroencephalography) approach. NF learning is a complex phenomenon and a controversial discussion on its feasibility and mechanisms has arisen in the literature. Critical aspects of the design of $\mathrm{FMRI-NF}$ studies include the localization of neural targets, cognitive and operant aspects of the training procedure, personalization of training, and the definition of training success, both through neural effects and (for studies with therapeutic aims) through clinical effects. In this paper, we argue that a developmental perspective should inform neural target selection particularly 
for pediatric populations, and different success metrics may allow in-depth analysis of NF learning. The relevance of the functional neuroanatomy of NF learning for brain target selection is discussed. Furthermore, we address controversial topics such as the role of strategy instructions, sometimes given to subjects in order to facilitate learning, and the timing of feedback. Discussion of these topics opens sight on problems that require further conceptual and empirical work, in order to improve the impact that fMRI-NF could have on basic and applied research in future.

Keywords

BCl; Developmental Cognitive Neuroscience; Multivariate pattern analysis; real-time fMRI-based neurofeedback; Translational research; Review

\section{Introduction}

The last decade has seen significant increase in the number of research groups using real-time, functional magnetic resonance imaging-based neurofeedback (fMRI-NF) to train participants in the self-regulation of brain networks and functions (Sulzer et al., 2013; Thibault, Lifshitz, \& Raz, 2018). NF utilizes the latest developments of real-time data processing and pattern analysis to train participants in the self-modulation of neural networks (LaConte, Peltier et al. 2007, Johnston, Boehm et al. 2010, Johnston, Linden et al. 2011, LaConte 2011, Watanabe, Sasaki et al. 2017). The strength of this technique, compared to approaches with EEG (Arns, Batail et al. 2017) or functional nearinfrared-spectroscopy (fNIRS) (Sitaram, Ros et al. 2017), lies in its high spatial resolution, the ability to probe deep subcortical structures and whole-brain coverage, as well as the extraction of information from distributed activation patterns (Sorger, Reithler et al. 2012), and the mapping of functionally connected networks (Zotev, Krueger et al. 2011). This is critical, as functional brain networks change significantly throughout development (Cohen Kadosh, Cohen Kadosh et al. 2011) and the fine-tuning patterns differ for typically and atypically developing populations (Johnson, Halit et al. 2002, Cohen Kadosh, Linden et al. 2013). If implemented successfully, the NF approach holds much promise for brain-based intervention approaches that aim to influence and shape the emerging networks in the developing and matured brain. That is, it allows us to target not only cortical and subcortical task-relevant regions with good precision, but it also offers the necessary flexibility to accommodate the frequent changes in brain network configurations that are typical for emerging networks. As a result, a substantial body of "neurofeedback training" research is now available that highlights the potential and versatility of feedback-based approaches to teach participants to control and improve brain functions.

Operant conditioning is widely acknowledged as the leading learning mechanism of brain selfregulation with NF (Black et al., 1977; Caria, 2016), which is mediated by reinforcement of action that results in a desired change in brain activation. The two-process theory (Gaume et al., 2016; Lacroix, 1986) adds a second, parallel process, which focuses on discrimination learning of internal percepts, guided by the identification of correlation between internal (e.g. afferent signals, mental events, affective states) and external signals (i.e. neurofeedback). The integration of internal percepts with NF relies on the allocation of attention to the different sources of these signals, which is moderated by executive function (Gaume et al., 2016). The participant perceives reinforcing feedback and evaluates it with regards to the regulation goal. ). If accessible to perception, the subject improves his or her perceptual accuracy of internal signals. Depending on the valuation outcome, mental actions such as cognitive strategies are then changed or maintained (Paret and Hendler, under reviewA detailed overview of NF learning theories can be found here (Arns et al., 2017; Gaume et al., 2016; Sherlin et al., 2011; Strehl, 2014). 
With the advent of fMRI-NF, the number of specific brain mechanisms and indications for NF training has broadened considerably (Sulzer, Haller et al. 2013). The functional specificity of fMRI might explain the versatility of $\mathrm{fMRI-NF}$, showing modulation effects across a number of domains such as motor function (Sitaram, Veit et al. 2012, Scharnowski, Veit et al. 2015), prosody (Rota, Handjaras et al. 2011), working memory ((Zhang, Yao et al. 2013), visual sensitivity training ((Shibata, Watanabe et al. 2011, Scharnowski, Hutton et al. 2012, Sitaram, Veit et al. 2012), and emotions (Caria, Sitaram et al. 2010, Johnston, Linden et al. 2011, Zotev, Krueger et al. 2011, Paret, Kluetsch et al. 2014, Koush, Meskaldji et al. 2017) , for a review see (Linhartova, Latalova et al. 2019).

As a result, the number of studies on $\mathrm{fMRI-NF}$ has increased substantially and methodological progress has been considerable (Thibault, Lifshitz et al. 2018). We now have seen evidence that fMRI-NF training is feasible in participants across a wide age range, starting with children as young as seven years (Cohen Kadosh, Luo et al. 2016, Alegria, Wulff et al. 2017) and in a broad range of patient populations (see Thibault et al. 2018 for review), including prevalent psychiatric conditions such as chronic pain (deCharms, Maeda et al. 2005, Guan, Ma et al. 2015)), depression (Linden, Habes et al. 2012, Young, Zotev et al. 2014, Yuan, Young et al. 2014), borderline personality disorder (Paret, Kluetsch et al. 2016), posttraumatic stress disorder (Gerin, Fichtenholtz et al. 2016, Nicholson, Rabellino et al. 2017) and addiction (Li, Hartwell et al. 2013, Kirsch, Gruber et al. 2016). Evidence has also accumulated that $\mathrm{fMRI-NF}$ training affects responsiveness not only in the specific target region, but that it also modulates the underlying task networks (e.g., (Cohen Kadosh, Luo, et al., 2016; Keynan et al., 2016; Ruiz et al., 2013). Further, some studies have now begun to directly modulate brain networks. This has been achieved via functional connectivity (fc)-NF which has been used in both adults (Koush et al., 2017; Koush et al., 2013; Spetter et al., 2017), and adolescents (Jacob, Or-Borichev, Jackont, Lubianiker, \& Hendler, 2017; Zich, Luehrs, et al., unpublished data)). Finally, more recently, advanced methods such as multivariate pattern analysis (MVPA) and hierarchy indices between network's nodes (Jacob et al., 2017), have been introduced to assess distributed patterns of brain activation and functional correlations (Lubianiker, Goldway et al. in press).

While the technical aspects of fMRI-NF have been brought along to great success, which is reflected in advanced software developments and more scanning centers being now set-up to conduct fMRIbased NF experiments, the field finds itself at a crossroads which will not only determine whether the current research activity is sustainable in the future but will also have the impact that is hoped for with regards to interventions and clinical applications (Stoeckel et al., 2014). As with many newly emerging fields, it is important to verify that promises do not overstate the realistic possibilities. While the current evidence is certainly encouraging regarding the feasibility of fMRI-NF with a number of disease-relevant target areas and networks, it is now critical to tighten the strings and develop a methodological framework that can help guide future research, in particular on clinical applications. Furthermore, this will considerably facilitate the shift from feasibility and proof-ofconcept studies, which naturally dominate the initial phase of a research field, towards translational studies and clinical trials. Researchers developing NF training protocols cannot avoid to address fundamental questions regarding all steps of planning, conducting and analyzing; starting from the experimental design and leading all the way down to the evaluation of training success (Randell, McNamara et al. 2018). Broadly, critical questions can be sorted into four domains as they address (1) the neurocognitive function to be changed with the intervention; (2) the information given to participants by professionals beforehand and transmitted via the brain-computer interface; (3) personalizing the training context to the participant and (4) measuring brain self-regulation and training success. Addressed in this paper, these domains and the corresponding questions are visualized in the form of a Floor Map for graphical overview (Figure 1). The rapid development of NF 
techniques and applications, a large variety of suggested solutions for current problems, and the lack of gold standards are challenges that, in our view, require a debate considering several procedural aspects underlying the method. With this paper, we hope to contribute to the critical discussion of current progress in fMRI-based NF research and accordingly, to address the considerations and possible solutions the field is currently facing with. Though we focus on $\mathrm{fMRI}$, the large part of critical points stressed in this paper are not limited to NF with $\mathrm{fMRI}$, but pertain to any neuroimaging modality that can be used for this purpose, including EEG, fNIRS or magnetoencephalography (cf. (Thibault, Lifshitz et al. 2016, Sitaram, Ros et al. 2017). However, amongst all neuroimaging modalities, as fMRI-based NF is currently the most rapidly growing field (Watanabe, Sasaki et al. 2017), it also brings up certain aspects that require particular attention and justify focused review. Critically, by providing an overview on the different methodological, cognitive and psychological factors that influence NF training success, we hope to help steer the discussion towards improving and standardizing procedural aspects.

\section{How to choose the training target?}

\subsection{Target regions}

One of the most critical decisions facing fMRI-based NF research is without a doubt the choice of target and control region(s). Among others this involves the question whether fMRI-based NF should focus on local activation in a specific target region (typically a change in average blood oxygenation level dependent [BOLD] response), or rather metrics related to a network function or organization. Either approach has been shown to be feasible, yet a couple of theoretical aspects need to be considered.

If a single target region is chosen to be modulated with NF training, it is important to consider whether the main goal of the training is to regulate activation in a region that is disturbed, such as for example modifying amygdala BOLD activity in an emotion regulation context (Zotev, Krueger et al. 2011, Linden, Habes et al. 2012, Paret, Kluetsch et al. 2014), reducing ventral striatum reactivity to alcohol cues (Kirsch, Gruber et al. 2016), or in a region that is associated with increased food cravings (Ihssen, Sokunbi et al. 2016). The regional approach has governed the fMRI-NF practice, led by the assumption that some regions might be critical for a specific symptom or disorder. Yet, the critical issue here is to use a region that is vastly connected and could be considered as a hub, assuming that its modulation will result in a large functional impact through its network (Table 1 ).

It has become increasingly accepted among neuroscientists that functionally inter-connected networks of regions mediate brain processes (Broyd, Demanuele et al. 2009, Bullmore and Sporns 2009, Menon 2011, Barrett and Satpute 2013) especially regulatory ones (Kober, Barrett et al. 2008, Raz, Winetraub et al. 2012). According to this view, communication between and within largely segregated neural modules is essential to the transfer and processing of information in the brain, and it would make sense to target parameters of this communication with NF. One possible network metric that can be probed with NF is functional connectivity (fc) between regions, aiming to differentially change the co-activation patterns between two or more regions (Watanabe, Sasaki et al. 2017). This approach is possibly the more physiologically valid given that the brain is naturally organized in networks of regions that work together to support behaviour (Fair, Dosenbach et al. 2007, Broyd, Demanuele et al. 2009, Fair, Cohen et al. 2009, Cao, Wang et al. 2014). It is, however, important to recognize that network NF approaches should not neglect the behaviour of single brain regions within that network. Thus, for example, it can be assessed whether a specific brain region within the network is driving the modulatory change, as shown in a study which used fc-NF to change effective connectivity between the prefrontal cortex and the amygdala in an emotional 
reappraisal context (Zich, Haller, et al., unpublished data). In this study, Zich and colleagues found that fc-NF modulated the prefrontal cortex (PFC)-amygdala fc towards a more negative fc pattern, and that this modulation was driven predominantly by changes in PFC activity. The authors also found that this fc change, which is related to more control and less anxiety correlated with the change in thought control ability as assessed before and after the MRI session.

\subsection{Consider functional neuroanatomy of neurofeedback learning}

In a recent paper, Sitaram and colleagues presented three different NF brain network models that support the overall NF process (Sitaram, Ros et al. 2017). Focusing on key brain regions that have been repeatedly shown to be activated in previous NF studies, they differentiated between network regions associated with control of visual NF, such as the dorsolateral PFC (dIPFC), thalamus, lateral occipital cortex and posterior parietal cortex, NF learning regions such as the dorsal striatum, and finally, NF reward processing regions, such as the ventral striatum, the anterior cingulate cortex (ACC) and the anterior insula. Moreover, neural activations related to NF control and those related to the monitoring of feedback such as reward signals could be recently differentiated (Paret, Zahringer et al. 2018). In this study, healthy individuals viewed emotional pictures and regulated their amygdala response, visualized via the temperature of a thermometer presented next to the pictures. The task involved two conditions, requiring individuals to regulate NF either up or down. That is, they needed to evaluate NF with respect to the task-condition (e.g. rise in temperature is rewarding during up-regulation but punishing during down-regulation). In line with Sitaram et al.'s model, the dIPFC, lateral occipital cortex, and lateral thalamus were involved in NF control, whereas the ventral striatum was involved in reward processing. This finding aligns well with other empirical study of NF-related reward signals (Ramot, Grossman et al. 2016, Shibata, Lisi et al. 2019, Skottnik, Sorger et al. 2019). In contrast to Sitaram et al.'s suggestion, the insula was not involved in reward processing but rather in NF control (Shibata et al. 2019, Paret et al. 2018). In addition, the ventromedial PFC (vmPFC) was involved in feedback monitoring (Radua, Stoica et al. 2016, Paret, Zahringer et al. 2018, Shibata, Lisi et al. 2019), as were the medial thalamus and the rostral PFC (Paret et al. 2018). Notably, these regions did not differentiate between rewarding or non-rewarding feedback, whereas the orbitofrontal cortex was found to respond to failure feedback selectively (Paret et al., 2019). Instead, the vmPFC and medial thalamus exhibit a more general feedback response, suggesting a role in attentional control. The vmPFC may also support the learning of associations between emotional arousal and feedback ((Paret, Ruf et al. 2016); see also (Radua, Stoica et al. 2016) for vmPFC response to NF). In contrast, the rostral PFC showed a marked response when subjects were instructed to up-regulate feedback but not during down-regulation (Paret et al. 2018). A positive correlation with regulation success and functional connectivity between rostral PFC and ventral striatum further support a role of rostral PFC in the monitoring of contextual information such as instructions (Paret et al. 2018). Rostral PFC resting-state connectivity predicted anxiety reduction with fMRI-NF (Scheinost, Stoica et al. 2014), a finding that further emphasizes the importance of this neural node for the ability to effectively process feedback in order to inform brain self-regulation. Involvement of rostral PFC may therefore reflect effortful model-based reinforcement learning of regulation strategies (Gaume et al. 2016).

Brain self-regulation with NF is an active task and involves interlocked psychological processes. In addition to regions involved in feedback monitoring, regions involved in active NF control show great overlap between studies (Emmert, Kopel et al. 2016). The network comprises regions from the cognitive control network including lateral PFC and posterior parietal cortex (Gaume et al., 2016), the anterior cingulate cortex (ACC) which is assumed to implement error monitoring of NF (Gaume et al., 2016), insula and lateral thalamus (Emmert, Kopel et al., 2016, Paret et al. 2018). As these 
regions are activated when subjects try to control NF, down-regulation training with these regions seems incompatible with the psychobiological dynamics of the system. In line with this assumption, Veit et al. (Veit, Singh et al. 2012) showed that down-regulation of anterior insula activation was associated with lower activation compared to up-regulation, but insula was activated above baseline in both conditions. Instead of down-regulation below baseline, alternating up- and down-regulation of dIPFC can increase speed of deliberate dIPFC regulation and may lead to more flexible recruitment of cognitive control (Van den Boom, Jansma et al. 2018). Feeding back activation from regions while they are themselves involved in NF may introduce problems for learning regulation, and should be considered in study planning (Lubianiker et al., in press). Taken together, several neural networks are involved in NF monitoring and control and feeding back activation from these networks may interfere with the actual treatment goal.

\subsection{Control aspects}

In order to show specificity of training, studies introduce control groups that, instead of feedback from target brain activation, receive some other kind of intervention or feedback protocol. The most commonly adopted choice of feedback control is to feedback signal stemming from a different brain region (other approaches include: no feedback; feedback based on the activity from the same region, but from a different point in time; feedback from the same region, but from a different individual; feedback based on artificially created irrelevant randomized signals, also sometimes called sham feedback). Here, similar concerns apply to the choice of a region from which the participant receives sham feedback in order to assess the sensitivity and specificity of the NF training. Generally, the control feedback region should be independent of the NF network that is to be trained. Given that most cognitive and behavioral functions depend on intertwined networks, this is a challenge that has yet to be addressed satisfactorily(Cohen Kadosh, Linden et al. 2013, Cohen Kadosh, Lisk et al. 2016, Staunton and Cohen Kadosh 2019)). Recently published taxonomies (Sorger, Scharnowski et al. 2019, Lubianiker, Goldway et al. in press) survey the advantages and disadvantages of the different ways of designing control conditions and selecting control groups. For example, not involving a control group or comparison to a no-training control group allow slim and less costly study designs, and are favorable in early phase studies (e.g. to identify endpoints for future clinical trials, (Sorger, Scharnowski et al. 2019)). If NF is given in order to facilitate mental strategy learning, a control group practicing a mental strategy without NF is necessary to reveal additive effects from treatment (Sorger et al. 2019). In contrast, placebo control is required to conclude on causality of brain self-regulation (e.g. with a randomized control group design). Like placebos in pharmacology research, NF placebo conditions should control for psychosocial effects (e.g. attention by staff, receiving high-tech treatment, and so on) without altering the process targeted with the intervention (e.g. emotion regulation with amygdala neurofeedback). However, brain self-regulation with NF is a complex phenomenon and involves several processes itself such as perception, valuation of feedback, implementation of control and learning (see section 1.2 above). Therefore, placebo would need to invoke all these "NF general" processes, without addressing the target process (Lubianiker et al. in press). With current state of knowledge, an informed selection for placebo NF considering all the above points is difficult, if not impossible. A solution still to be tested may be "randomized ROI NF" (suggested in Lubianiker et al. in press), where different control regions-of-interest are assigned to participants of the control group. Moreover, ethical considerations may prevent usage of some control groups, particularly in patient treatment. For example, there is concern that sham feedback might encourage participants to abandon an otherwise successful strategy, simply because the feedback does not seem to support using it. Schabus (Schabus 2017) suggested that yoked feedback might introduce learned helplessness, with adverse consequences on motivation and learning with subsequent real feedback. Moreover, a 
recent study by Goldway et al. (Goldway, Ablin et al. 2019) provided additional support for the use of sham feedback after they observed subjective improvement after sham feedback in patients with chronic pain that faded in follow-up measurements.

Similarly, given the changes in recruitment patterns of different brain regions in the developing brain, it is likely that brain regions that are used at an earlier developmental stage are not necessarily relevant at a later stage (Johnson, Grossmann et al. 2009, Johnson, Jones et al. 2015). As a result of this, it would be extremely difficult to find a control brain region that would support a comparable function across different ages. A possible solution for this problem could be to provide authentic feedback during the regulation conditions and to compare the regulation success against a 'dry' regulation baseline (where feedback is not given). This would allow us to assess the extent to which participants rely on the live feedback signal to both learn and maintain their regulation strategies.

\subsection{Population aspects}

The selection of region(s), as well as other parameters, is of particular importance when training children with or without psychopathology (Cohen Kadosh, Linden et al. 2013, Cohen Kadosh, Lisk et al. 2016), although it does apply also to adults. For example, it is currently unclear whether one should teach young individuals to regulate brain responses that would support a cognitive function at the specific developmental stage, or, instead teach them directly to use the brain network that supports this ability in healthy mature adults (Figure 2). This may seem especially important if one considers the developing brain as an adaptive system, where the networks of brain regions that support cognitive abilities change interactively as a result of on-going brain maturation and cognitive development (Cohen Kadosh and Johnson 2007, Johnson, Grossmann et al. 2009). Hence, the temporary use of an alternative brain network during development can be considered both logical and adaptive and it remains to be determined whether NF should target these current alternatives instead of future key regions. This is also relevant for other clinical populations (e.g. stroke patients), where brain networks may well have specialized in a way that is most adaptive for the individual. This has not only theoretical and practical, but also ethical, implications. More precisely, the main choice lies between increasing responsiveness in compensatory networks, or in attempting to shape brain networks towards more typical functioning. While either approach could be argued for, a definite decision should be best based on scientific evidence of mechanisms of plasticity and rehabilitation that is currently lacking. One promising solution may be to focus on brain regions that help with acquiring new skills, such as the inferior frontal gyrus for example, whose involvement in executive functions makes it a frequently reported brain region in developmental neuroimaging studies that observe age-dependent differences in brain activation (Johnson, Grossmann et al. 2009, Cohen Kadosh, Johnson et al. 2013).

\section{How to guide the trainee?}

\subsection{Feedback interface}

Interfaces of different complexity have been applied, ranging from simple, thermometer-like displays (Cohen Kadosh, Luo et al. 2016) to more complex reality interfaces (Yamin, Gazit et al. 2017). Integrating realistic virtual environments in NF can enhance learning and improve user's experience ((Cohen, Keynan et al. 2016), for discussion see (Lubianiker, Goldway et al. in press)). Yet, complexity can also prove to be distractive as it introduces additional attentional task demands, especially in pediatric or clinical populations which may present with reduced cognitive functions ('entertainment' vs. 'treatment' problem, see (Arns, Heinrich et al. 2014)). The optimal feedback modality (e.g., visual, auditory, tactile) and the complexity of their transmission (one dimensional 
courser movement to virtual reality environment) have to be chosen based on the objective, population and task (Table 2).

\subsection{Instruction}

Previous NF studies have varied as to whether participants were given explicit strategy instructions to regulate their brain responses or whether an implicit strategy was expected, i.e., with participants being asked to develop their own effective strategies. Most fMRI-NF studies to date have opted for implicit strategies (e.g. (Paret, Kluetsch et al. 2014)), and only some patient studies suggested the use of mental imagery (e.g. (Zilverstand, Sorger et al. 2015)). Others have only offered simple and relatively unconstrained instructions, in order to allow participants across a wide age range (7-17 years) to perform well. This was the case in a recent NF study where children and adolescents were asked to increase the response in a key emotion regulation region by trying 'to think happy thoughts; to think about something that would make them happy' (Cohen Kadosh et al., 2016). Keeping instructions simple may also be important for patient populations, when ability levels to use different strategies are likely to vary significantly. The same study also found that whereas the instruction worked in the first instance, this lasted only for a couple of runs, with some participants failing to up-regulate in the later runs. Understanding the motivational-contextual factors that modulate NF regulation is critical. Identifying individual differences in the use of different regulation strategies is also important, if we want to enhance the effectiveness of these procedures. In fact, a collection of post-training feedback from subjects across experiments may be helpful with identifying any trends in strategy formation/usage.

NF can also be used to support the selection of an instrumental mental strategy from a pool of potentially useful strategies (Lawrence, Su et al. 2014). Patients can identify effective strategies for ACC control from a pool of skills they had previously learned during cognitive behavior therapy treatment (MacDuffie, Maclnnes et al. 2018). Mental strategies during training are diverse and depend on the NF signal; for example, motor imagery can be used to control sensorimotor electrocortical rhythms (Halder, Agorastos et al. 2011, Nan, Rodrigues et al. 2012)and cognitive appraisals of emotional contents are instrumental for amygdala regulation (Brühl et al., 2014; Zotev et al., 2011). It was shown that strategy knowledge can facilitate NF learning (Bray, Shimojo et al. 2007, Lawrence, Su et al. 2014, Zilverstand, Sorger et al. 2015, Kober, Witte et al. 2017), and strategy instructions may decrease the risk of non-learning with limited duration of an experiment (Scharnowski and Weiskopf 2015) or in face of erroneous control beliefs, as shown in EEG-NF (Witte, Kober et al. 2013). Furthermore, practicing anterior insula up-regulation with NF helped subjects to identify helpful mental strategies, while subjects repeating strategies without NF did not learn anterior insula regulation (Caria, Sitaram et al. 2010).

On the downside, strategies can have undesired effects. For example, when subjects identify an instrumental strategy right away, there is no need to explore new strategies, which would potentially lead to even stronger activation. Some support for this critique of strategy suggestions comes from EEG-NF research, where Hardman et al. (Hardman, Gruzelier et al. 1997) observed a steeper learning curve in subjects without instructed strategies to control feedback from slow cortical potentials, compared to another group of subjects who were told to use emotional strategies. However, significant post-hoc tests were not reported to show whether the no-strategies group in the last of three blocks outperformed subjects from the instructed-strategies group. In line with a possible advantage not to instruct strategies, strongest learning effects were observed for fMRI-based NF without strategy instruction, followed by NF with strategy instruction; but group differences were not significant (Sepulveda, Sitaram et al. 2016). These results need a careful interpretation, because the authors of the latter study introduced another variable (additional 
rewards) and statistical tests of group differences may have lacked adequate power. Though these studies lend some support to the notion that strategy instructions may (negatively) influence NF regulation learning, these results may also indicate that subjects were able to overcome initial ignorance of strategies to regulate NF, while subjects who received strategy instructions start with a higher level of regulation success, which they maintain over the course of the experiment. Evidence has now accumulated showing that explicit strategy knowledge is not required for acquisition of NF control per se (Shibata, Watanabe et al. 2011, Kober, Witte et al. 2013, Amano, Shibata et al. 2016, Koizumi, Amano et al. 2016, Ramot, Grossman et al. 2016, Shibata, Watanabe et al. 2016) for a review see Shibata et al. 2019). Nonetheless, instructed strategies may be useful when using NF for certain applications, e.g. emotion regulation purposes. For instance, individuals who habitually use specific emotion regulation strategies, such as the reappraisal of emotional material, have better functioning levels and higher well-being compared to others, who tend to suppress emotions (Gross and John 2003). Reappraisal is a cognitive strategy to change the emotional impact of an affective stimulus via re-interpretation of, or taking a detached perspective from the stimulus (Powers and LaBar 2019). Healthy individuals prefer to distance from emotional stimuli with high affective intensity, but rather reappraise low intensity stimuli (Sheppes, Scheibe et al. 2011, Sheppes, Scheibe et al. 2014). Deviations from this pattern may relate to psychopathology, such as borderline personality disorder (Sauer, Sheppes et al. 2016). Hence, NF with explicit strategy instructions may help patients to overcome deficits in the use of effective emotion regulation strategies, through neural reinforcement of desirable mental strategies (Herwig, Lutz et al. 2019). The question of whether explicit or implicit task instructions are most efficient remains to be determined, yet given the importance of this methodological aspect (Birbaumer, Murguialday et al. 2008), the need for a more systematic research and possible standardization is evident.

\section{How to personalize the training?}

Based on the questions above, it has also become clear that methodological approaches may need to be additionally adapted for different populations, as requirements are likely to vary not only across different ages or clinical populations, but also with regard to subject-specific psychological variables which we will discuss in the next section. There are a number of cognitive and psychological factors that can affect NF performance. Gaining a better understanding of these factors is not only important in order to improve training outcome but also to help address the inefficiency problem. The inefficiency problem refers to the often reported finding that not all participants in NF studies are able to influence their brain activity. These people are often referred to in the literature as non-responders, non-performers or non-regulators and represent $30-50 \%$ of the population (Alkoby, Abu-Rmileh et al. 2018). In the following, we discuss a number of methodological, cognitive and psychological factors that all contribute to NF efficiency. While by no means complete, these provide first starting points for further optimizing NF interventions for a specific sample and thereby reducing the inefficiency problem.

\subsection{Protocol aspects}

An area of NF research that requires more in-depth research is the development of both time and cost-effective training regimes. For example, the number and length of each training session varies considerably across the different studies, with some studies testing participants repeatedly over several days (e.g., (Spetter, Malekshahi et al. 2017)) whereas others used a single testing session only (e.g., Cohen Kadosh, Luo, et al., 2016)). Some individuals might achieve the maximum level of control already after a single session, notably not only in fMRI-NF but also in EEG-NF (Schabus 2017, Schabus, Griessenberger et al. 2017, MacDuffie, Maclnnes et al. 2018). The former is surprising as EEG-NF is usually thought to require higher training dosage than fMRI-NF (Sulzer, Haller et al. 2013). 
The lack of reported data on within-session brain signal regulation is currently a major obstacle for comprehensive review (Thibault and Raz 2016). A more systematic investigation of optimal training length is urgently needed to avoid training regimes that are too short, or too long to be effective. To date, only one study has considered individual criteria for training length (Scharnowski, Hutton et al. 2012). More research, across a range of populations and tasks would now be needed to provide reliable standards for effective and efficient NF training. Similarly, when considering training length and intensity, the option of combining NF training with out-of-scanner practice should be considered, as has been done in some studies with clinical samples (Subramanian, Hindle et al. 2011, Linden, Habes et al. 2012), possibly in combination with a therapeutic intervention, such as cognitive-behavioral therapy (CBT), which would allow for a cross-over and mutual reinforcement of intervention approaches. NF could augment psychotherapy as illustrated by MacDuffie et al. (MacDuffie, MacInnes et al. 2018), who let patients regulate ACC after completion of CBT, using strategies they had learned in psychotherapy. The one-session protocol was instrumental for patients to identify strategies that were more effective than others for neural regulation. Furthermore, Herwig et al. (Herwig, Lutz et al. 2019) show that NF may augment the neural effect of behavioral instructions.

Finally, a number of contextual and individual factors such as at what time of the day participants are tested, age, the amount of sleep that the participant had, physical exercise and general fitness, nutrition and menstrual cycle could influence the optimal training regime. At this time, we have no data available to address these questions conclusively. In addition to finding an ideal NF protocol, it might be helpful to try and implement an adaptive NF protocol that could maximizes neural regulation abilities. One example of such an approach to support optimal regulation performance could involve the individual NF range to keep participants challenged and engaged by varying scales of neural activity representation via the interface. Adaptive task difficulty was previously applied in other types of tasks, such as the tracking stop task (Rubia, Smith et al. 2003) by using a computer algorithm that adjusts the paradigm difficulty level according to the participant's performance. Using this approach, feedback range would not be fixed for all participants, rather it will be set according to the participant's performance in previous blocks/sessions. Such "shaping" of behavior conforms to learning theory principles (Sherlin et al., 2011). For example, if the participant was very successful in previous sessions, the feedback will be presented with a higher demand range of neural activity in order to achieve the optimal performance.

Another approach to maximize regulation performance is the use of adaptive NF protocols that contain fewer challenge levels. According to this approach, individual progress in NF training is determined by the participant achieving a pre-determined goal, rather than by going through a fixed number of sessions. This kind of protocol may include fewer stages, each carrying different level of challenge. The first stage of such NF protocol may include a neutral feedback interface (e.g. bar/neutral sound) while more advanced stages may include gradually more challenging (i.e., stressful or intriguing) feedback interfaces. In this setup participants are required to meet predetermined success criteria (e.g. three successful sessions), in order to progress from one stage to the next (see Table 4 for an overview). Whilst adopting an individually tailored feedback approach may be more optimal from a learning perspective, it significantly limits comparability between sessions (either within or between individuals), and in turn reduces the informative value of, especially, between-subject designs.

\subsection{Person aspects}

Cognitive factors such as general intelligence levels, executive functions and the ability to focus on a task are to be considered when adjusting task designs for different populations (Hammer, Halder et 
al. 2012, Jeunet, N'Kaoua et al. 2016). It may be useful for example to simplify both task instructions and feedback display for younger participants in order to reduce the task demands and cognitive load (Gaume et al., 2016). A study by Alegria et al used a simple rocket to display brain activity in a sample of adolescents (Alegria, Wulff et al. 2017). In another recent study, Zich and colleagues trained a sample of adolescent girls to simultaneously increase activation in the dorsolateral prefrontal cortex and to decrease activation in the amygdala (Zich, Haller, et al., unpublished data), yet to simplify these complex changes for the participants, a simple thermometer was displayed, which increased with increasing negative correlation between the two regions.

A recent systematic review of the NF literature where 281 articles were reviewed (Staunton and Cohen Kadosh 2019) found that attention indices such as the ability to focus, as well as length of attention span influenced NF learning outcomes, yet more research needs to be conducted to explore this further.

The contribution of psychological factors has been less explored and the results so far are not conclusive. In the recent systematic review mentioned above, only two factors emerged: motivation and mood (Staunton \& Cohen Kadosh, 2019). Understanding a participant's motivational preference is important because it can help explain how participants will respond to the training paradigm. However, motivation is likely to vary between and within participants, but also as a function of age and across contexts. Specifically, it was found that while motivation to learn is important, there may be a need to balance individual levels of intrinsic motivation with the reward that is received. For example, Leeb and colleagues (Leeb, Lee et al. 2007) found that whereas highly motivated participants performed initially better on a simple NF training task than less motivated students, this performance difference was reversed when the NF training paradigm was switched to a virtual environment and became more immersive and hence, more interesting for subjects. This suggests that external factors such as learning paradigms can interact with an individual's motivation to learn. With regard to mood, it has been shown that anxiety and depression can have a negative influence on performance (Zich, Haller, et al., unpublished data). In a recent study, Zich and colleagues found that low state anxiety levels in adolescent girls at the beginning of the training predicted better NF training outcomes (Zich, Haller et al., unpublished data). While more research is needed to replicate this finding, future studies could explore the use of anxiety reducing exercises prior to the NF training in order to maximize its effectiveness. Similarly, it may be useful to monitor anxiety levels in participants throughout the training and to adjust when a certain threshold is reached, rather than continuing with the training.

Further, being susceptible to other people's emotion appears to play a part in emotional regulation learning. For example, susceptibility to anger has been found to impair NF success in two studies (Zotev, Krueger et al. 2011, Marxen, Jacob et al. 2016). Moreover, under specific circumstances, the ability to label emotions may be important in NF-based emotion regulation learning, such as in the case of children and adolescents who exhibit prolonged developmental trajectories for both emotion recognition and regulation abilities (Thomas, De Bellis et al. 2007, Ahmed, Bittencourt-Hewitt et al. 2015). Introspective ability to perceive one's emotions more generally relates to the ability to discriminate percepts of internal signals, which is a key aspect of the two-process theory of NF learning. As suggested by Gaume et al. (2016), the ability of participants to perceive internal signals may be a good predictor for learning success and transfer of learning. However, behavioral measures of the ability to perceive brain states are still to be developed.

The evidence reviewed above highlights the important role that psychological factors can play in the successful implementation of NF training (see also Staunton \& Cohen Kadosh, 2019). In the same vein, neural markers were found successful to predict EEG-NF performance (Gevensleben, Holl et al. 
2009, Blankertz, Sannelli et al. 2010) and could be distilled from pretraining fMRI measures such as resting-state connectivity (Scheinost, Stoica et al. 2014) to predict NF response and optimize treatment protocols. Clearly, more research is needed to develop recommendation that would allow us to personalize training programs for each individual in order to achieve maximum effectiveness.

\section{How to monitor training?}

\subsection{Signal calculation}

There is currently no standardized approach of calculating feedback, a problem which makes it difficult to compare NF signal and, in turn, NF training effectiveness across studies. To quantify signal change, BOLD signal drifts require corrections such as signal detrending and/or usage of a baseline where signal drifts only have negligible influence. For example, baseline activation can be retrieved from an epoch preceding the regulation block such as a 'rest'-period of a few seconds where subjects are instructed to refrain from cognitive efforts (Veit, Singh et al. 2012), and activation can be quantified relative to the mean baseline activation. BOLD signal changes have considerable interpersonal and intrapersonal variance that renders quantification problematic, though. To deal with this problem, the BOLD signal can be sampled during a baseline period (e.g. $60 \mathrm{~s}$ ) to receive the mean and standard deviation, which can then be used as reference for quantification of signal variability (e.g. (Keynan, Meir-Hasson et al. 2016)). This method seems optimal to receive maximally informative feedback in terms of variability, but a sufficiently long baseline period needs to be recorded in order to receive reliable statistics. Notwithstanding the problems of BOLD quantification, absolute quantification of BOLD signal change for feedback appears feasible (e.g. Paret et al., 2014, Zotev et al., 2011), at least for some applications (Figure 3). Another question regards the specificity of BOLD signal changes. First, the BOLD signal is prone to changes in blood oxygenation that are not related to neural activations, for example produced by changes in respiratory patterns, which need adequate control (see Thibault et al., 2018 for a comprehensive review). Second, although most NF studies use some sort of artifact correction to control for noise, few studies tried to confirm specificity of feedback. It is usual practice to carefully select the anatomical location of a region of interest via functional localizer or anatomical masks. However, the $\mathrm{fMRI}$ signal of the whole brain is considerably inter-correlated and only few studies analyze correlations of the feedback signal with brain-wide activations post-hoc. For instance, Ramot et al. (2016) state that feedback-correlated brain activation was indeed not confined to the target region in the fusiform face area, but was instead widespread and included regions such as thalamus, cerebellum and posterior cingulate cortex. In a recent study, we recorded background noise from a rectangular control region located few millimeters from the amygdala, and subtracted the signal from the right amygdala's BOLD signal. A post-hoc whole-brain regression analysis with feedback as predictor showed strongest correlation of the target region, while left amygdala and two regions in the right and left cerebellum were also correlated (Paret et al., 2018). In conclusion, though feedback is given from a region of interest, the feedback signal is likely not restricted to the anatomical boundaries drawn by the experimenter. Instead, feedback more or less carries information on activation from other regions throughout the brain, beyond the a priori selected target region. In cases of increasing co-activation, feedback becomes more representative for neural network activity. Conceptually, the difference between regional and connectivity feedback is blurred under these circumstances (Figure 4). Notwithstanding efforts to yield anatomically precise signal, it is even not clear whether an NF signal with high anatomic specificity (e.g. from the right amygdala only) is superior to a less localized signal. It is even possible that the latter is more helpful for regulation learning, as a less anatomically restricted signal may be more representative of functional networks of interconnected brain regions, and provide greater ecological validity. In future, studies could clarify this matter by providing whole-brain maps of feedback-correlated brain activation in 
addition to regulation success indices, and with verbal feedback from subjects about the perceived regulation success.

Feedback can be presented continuously or with a delay, and both approaches have been shown to be feasible in allowing participants to learn and to modify their brain response. For some feedback modalities such as dynamic causal modeling (Koush, Meskaldji et al. 2017), continuous feedback is not feasible and intermittent/end-of-block feedback turned out to be sufficient for NF training. Only few studies compared continuous and intermittent feedback, and evaluated effects on neuromodulation. Results are inconsistent: two groups stated that continuous feedback was superior for premotor cortex regulation (Johnson, Hartwell et al. 2012) and amygdala regulation (Hellrung, Dietrich et al. 2018), while others tentatively suggest better auditory cortex regulation with continuous feedback (Emmert et al., 2017). In fact, two of the studies did not find significant differences between both types of feedback in primary data analyses (Emmert et al., 2017, Hellrung et al., 2018), and the other study did not report the required statistical group comparison (Johnson, Hartwell et al. 2012). Thus, no clear evidence is currently available on the superiority of continuous or intermittent feedback. Better knowledge of the functional neuroanatomy underlying brain selfregulation could inform decision of continuous vs. intermittent. For example, if the target region shows an event-related response to feedback, it is probably better to give feedback intermittently, because otherwise, NF monitoring may interfere with NF control (Lubianiker et al., in press).

\subsection{Success metrics}

The ultimate question for brain self-regulation training is whether the training was successful or not. However, there is currently no established practice of how success is measured. A number of approaches have been taken so far. Some studies have looked at the number of successful trials (Chiew, LaConte et al. 2012, Koush, Rosa et al. 2013) an effect size of up/down regulation (Cohen et al., 2016) or a combined index which compared regulation vs. rest activity in the target regions (Cohen Kadosh, Luo, et al., 2016; Zotev et al., 2011). Further considerations are whether success is measured by changes in the NF training sessions only or at a follow-up testing appointment. Alternatively, success can be operationalized as improvements in related tasks, i.e. behavioural transfer effects. One could also look at subsequent functional changes in the brain, such as changes in local or global connectivity (Shibata, Watanabe et al. 2011, Zotev, Phillips et al. 2013, Cohen Kadosh, Luo et al. 2016). Similarly, training effects could be observed at the structural brain level, i.e. in change in white or grey matter.

Assessing NF training success will also depend on the statistical approach. The optimal approach will depend on whether the training consists of a single session or multiple sessions. For a single session, a simple t-test or Wilcoxon t-test ("fixed threshold") can be used to compare activation during a passive baseline condition (e.g. where subjects were instructed to 'rest') and regulation conditions (Figure 5A), or between NF-based regulation during exposure to visual stimuli and a "mirror run" (passive viewing of the same/ matched stimuli) (Ihssen, Sokunbi et al. 2016). However, whereas this measure is straightforward, it is also quite insensitive as it provides binary yes/no answers regarding the question if the NF session was successful or not (Krause, Benjamins et al. 2017). Moreover, global changes in brain signals during training (see above) might mask the true difference between 'rest' and 'regulate' conditions. To overcome this issue, one can perform the same statistical test but with one difference: instead of comparing average brain activity during 'rest' to average brain activity during 'regulate', compare each regulation condition to the previous rest condition. In this case, instead of having a binary score for the session, one can constitute a more fine-grained type of measure we call "success rate" (Figure 5B). However, this measure is not continuous, as it depends on the number of NF blocks in each session, thus provides fixed levels of success that preclude 
parametric testing. To overcome the lack of continuity, one could simply calculate the difference (delta) between the mean activity levels in the rest and regulate conditions. In addition to adding continuity, this measure allows for a straightforward interpretation of the results. There are several drawbacks however, as this measure is highly noise sensitive and affected by outliers. Furthermore, it overlooks differences in within-session variance of the signal. For example, while two participants may have equal delta they could differ in the signal variance. Thus, the same delta might not reflect equal performance. One possible solution is the use of a "personal effect size" that divides the "delta" by the pooled standard deviation of 'rest' and 'regulate' conditions (Figure 5C). This measure is continuous and accounts for differences in individual variance. However, this measure is quite sensitive to the block duration and sampling rate - with greater sampling rate leading to a reduction in variance, thereby making it difficult to compare different designs and interfaces (for a summary see Table 4).

In order to account for global performance throughout the NF training course, there is a need to create an index that would capture NF performance not only during a single session but across the entire training procedure. There are currently few approaches to address this issue: the first option is to calculate the "mean performance", i.e. an averaged success index across sessions. This index assesses the participant's performance in a global manner throughout the NF training and is relatively noise-insensitive. One problem with this approach is that the mean performance index does not take into account whether the participant's performance improves from one session to the next. In order to account for any change in regulation ability, one can use the difference between the first and last session. However, this would only provide an index of change from the first to the last session, which would mask any change throughout the training day, a problem which is more obvious when we consider that some participants will exhibit their best performance during the intermediate sessions of the training, followed by a decrease due to tiredness or boredom (Cohen Kadosh, Luo, et al., 2016). One way to access this additional information is to calculate the "learning slope", i.e. the slope of a regression of success index on session number. While this measure may account in the best way for learning dynamics, it suffers from two major drawbacks: first, it assumes that learning follows a linear trajectory, which is an assumption that is not supported in current learning theories. For example, skill learning theory for NF learning predicts rapid initial change in performance and more moderate improvement at later phases (Yin, Mulcare et al. 2009, Sitaram, Ros et al. 2017). Secondly, this measure is noise sensitive, meaning that one failed NF session can affect the overall model fit. An additional measure that has different conceptual framing is "best performance". This measure refers only to the best session in the NF training course and might even refer to the best block. The rationale underlying this measure is that it reflects the subjects' best potential of neural regulation. With that, this measure is highly sensitive to noise but prone to outliers (see Table 4). Altogether, it seems that selecting success indices based on theoretical considerations constitutes a helpful initial starting point. We now urgently require systematic research into the mechanisms that relate to changes in NF learning indices and neural/behavioral outcome measures.

\section{Conclusion}

Researchers about to design and conduct an NF experiment need to find answers on a variety of questions for which clear guidelines are missing. In the above, we addressed several questions regarding NF procedures and reviewed the current state of the literature. For the selection of brain targets, one should consider aspects such as developmental trajectories in brain development and the functional neuroanatomy of NF learning. Virtual reality interfaces have the potential to increase training efficiency by keeping subjects motivated yet the specific advantages over more simple 
interfaces that currently prevail require further study. Whether or not to provide overt strategies for $\mathrm{NF}$ regulation is still a controversial question and evidence for clear recommendations is lacking. There are virtually infinite ways to calculate NF signals. Control for nuisance and global brain signals is necessary for reliable NF, but the way in which the brain signal is "cleaned" from other influences can have unintended effects. Calculating both the absolute and relative quantification of BOLD signal change for $\mathrm{fMRI-NF}$ is feasible and suitable for training. Moreover, criteria for quantification of neural regulation success are lacking, as are success measures, which are necessary in order to evaluate training efficiency. Here we discuss different success metrics that may help to evaluate and compare training regimes in future. We also addressed options to personalize training regimes and to make training more effective, considering aspects of NF protocols as well as characteristics of persons undergoing training. Therefore, in order to maintain the current momentum in the field and the considerable progress as evident by the ever-increasing number of studies that are being published on NF research, a two-pronged approach will be needed. Specifically, we envision an active basic science approach which systematically strives to explore and optimize NF designs by investigating the effect of methodological, cognitive and psychological factors. Such an approach could also focus on establishing a comprehensive testing protocol that would enable us to compare NF training outcomes across populations and research centers. One crucial step towards this goal is adherence to reporting guidelines such as the CRED-nf (Ros, Enriquez-Geppert et al. pre-print) in future publications. This first approach would then inform the translational work in the field by providing authoritative guidelines for the design of more effective brain training for cognitive enhancement more generally, and the treatment of clinical populations more specifically.

\section{Acknowledgements}

CP received funding from the German Research Foundation (DFG PA 3107/1-1). KCK, TH and DEJL are supported by the BRAINTRAIN Collaborative Project, supported by the European Commission, under the Health Cooperation Work Programme of the 7th Framework Programme, under the Grant Agreement $n^{\circ} 602186$

\section{References}

Ahmed, S. P., A. Bittencourt-Hewitt and C. L. Sebastian (2015). "Neurocognitive bases of emotion regulation development in adolescence." Dev Cogn Neurosci 15: 11-25.

Alegria, A. A., M. Wulff, H. Brinson, G. J. Barker, L. J. Norman, D. Brandeis, D. Stahl, A. S. David, E. Taylor, V. Giampietro and K. Rubia (2017). "Real-time fMRI neurofeedback in adolescents with attention deficit hyperactivity disorder." Hum Brain Mapp 38(6): 3190-3209.

Alkoby, O., A. Abu-Rmileh, O. Shriki and D. Todder (2018). "Can We Predict Who Will Respond to Neurofeedback? A Review of the Inefficacy Problem and Existing Predictors for Successful EEG Neurofeedback Learning." Neuroscience 378: 155-164.

Amano, K., K. Shibata, M. Kawato, Y. Sasaki and T. Watanabe (2016). "Learning to Associate Orientation with Color in Early Visual Areas by Associative Decoded fMRI Neurofeedback." Curr Biol 26(14): 1861-1866.

Arns, M., J. M. Batail, S. Bioulac, M. Congedo, C. Daudet, D. Drapier, T. Fovet, R. Jardri, M. Le-VanQuyen, F. Lotte, D. Mehler, J. A. Micoulaud-Franchi, D. Purper-Ouakil, F. Vialatte and N. E. group (2017). "Neurofeedback: One of today's techniques in psychiatry?" Encephale 43(2): 135-145.

Arns, M., H. Heinrich and U. Strehl (2014). "Evaluation of neurofeedback in ADHD: the long and winding road." Biol Psychol 95: 108-115. Barrett, L. F. and A. B. Satpute (2013). "Large-scale brain 
networks in affective and social neuroscience: towards an integrative functional architecture of the brain." Curr Opin Neurobiol 23(3): 361-372.

Birbaumer, N., A. R. Murguialday and L. Cohen (2008). "Brain-computer interface in paralysis." Curr Opin Neurol 21(6): 634-638.

Black, A., Cott, A., Pavloski, R., 1977. The operant learning theory approach to biofeedback, in: Biofeedback, Theory and Research. Academic Press, USA, pp. 89-127.

Blankertz, B., C. Sannelli, S. Halder, E. M. Hammer, A. Kubler, K. R. Muller, G. Curio and T. Dickhaus (2010). "Neurophysiological predictor of SMR-based BCI performance." Neuroimage 51(4): 13031309.

Bray, S., S. Shimojo and J. P. O'Doherty (2007). "Direct instrumental conditioning of neural activity using functional magnetic resonance imaging-derived reward feedback." Journal of Neuroscience 27(28): 7498-7507.

Broyd, S. J., C. Demanuele, S. Debener, S. K. Helps, C. J. James and E. J. Sonuga-Barke (2009). "Default-mode brain dysfunction in mental disorders: a systematic review." Neurosci Biobehav Rev 33(3): 279-296.

Bullmore, E. and O. Sporns (2009). "Complex brain networks: graph theoretical analysis of structural and functional systems." Nature Reviews Neuroscience 10(3): 186-198.

Cao, M., J. H. Wang, Z. J. Dai, X. Y. Cao, L. L. Jiang, F. M. Fan, X. W. Song, M. R. Xia, N. Shu, Q. Dong, M. P. Milham, F. X. Castellanos, X. N. Zuo and Y. He (2014). "Topological organization of the human brain functional connectome across the lifespan." Dev Cogn Neurosci 7: 76-93.

Caria, A., R. Sitaram, R. Veit, C. Begliomini and N. Birbaumer (2010). "Volitional control of anterior insula activity modulates the response to aversive stimuli. A real-time functional magnetic resonance imaging study." Biol Psychiatry 68(5): 425-432.

Caria, A., 2016. Self-Regulation of Blood Oxygenation Level Dependent Response: Primary Effect or Epiphenomenon? Front Neurosci 10, 117. https://doi.org/10.3389/fnins.2016.00117

Chiew, M., S. M. LaConte and S. J. Graham (2012). "Investigation of fMRI neurofeedback of differential primary motor cortex activity using kinesthetic motor imagery." Neuroimage 61(1): 2131.

Cohen, A., J. N. Keynan, G. Jackont, N. Green, I. Rashap, O. Shani, F. Charles, M. Cavazza, T. Hendler and G. Raz (2016). "Multi-modal Virtual scenario enhances neurofeedback learning." Frontiers in Robotics and $\mathrm{Ai} 3$.

Cohen Kadosh, K., R. Cohen Kadosh, F. Dick and M. H. Johnson (2011). "Developmental changes in effective connectivity in the emerging core face network." Cereb Cortex 21(6): 1389-1394.

Cohen Kadosh, K. and M. H. Johnson (2007). "Developing a cortex specialized for face perception." Trends Cogn Sci 11(9): 367-369.

Cohen Kadosh, K., M. H. Johnson, R. N. Henson, F. Dick and S. J. Blakemore (2013).

"Differential face-network adaptation in children, adolescents and adults." Neuroimage 69: 11-20. 
Cohen Kadosh, K., D. E. Linden and J. Y. Lau (2013). "Plasticity during childhood and adolescence: innovative approaches to investigating neurocognitive development." Dev Sci 16(4): 574-583.

Cohen Kadosh, K., S. Lisk and J. Y. F. Lau (2016). "The Ethics of (Neuro) Feeding Back to the Developing Brain." American Journal of Bioethics Neuroscience 7(2): 132-133.

Cohen Kadosh, K., Q. Luo, C. de Burca, M. O. Sokunbi, J. Feng, D. E. J. Linden and J. Y. F. Lau (2016). "Using real-time $\mathrm{fMRI}$ to influence effective connectivity in the developing emotion regulation network." Neuroimage 125: 616-626.

deCharms, R. C., F. Maeda, G. H. Glover, D. Ludlow, J. M. Pauly, D. Soneji, J. D. E. Gabrieli and S. C. Mackey (2005). "Control over brain activation and pain learned by using real-time functional MRI." Proceedings of the National Academy of Sciences of the United States of America 102(51): 1862618631.

Emmert, K., R. Kopel, J. Sulzer, A. B. Bruhl, B. D. Berman, D. E. J. Linden, S. G. Horovitz, M. Breimhorst, A. Caria, S. Frank, S. Johnston, Z. Long, C. Paret, F. Robineau, R. Veit, A. Bartsch, C. F. Beckmann, D. Van De Ville and S. Haller (2016). "Meta-analysis of real-time fMRI neurofeedback studies using individual participant data: How is brain regulation mediated?" Neuroimage 124(Pt A): 806-812.

Fair, D. A., A. L. Cohen, J. D. Power, N. U. Dosenbach, J. A. Church, F. M. Miezin, B. L. Schlaggar and S. E. Petersen (2009). "Functional brain networks develop from a "local to distributed" organization." PLoS Comput Biol 5(5): e1000381.

Fair, D. A., N. U. F. Dosenbach, J. A. Church, A. L. Cohen, S. Brahmbhatt, F. M. Miezin, D. M. Barch, M. E. Raichle, S. E. Petersen and B. L. Schlaggar (2007). "Development of distinct control networks through segregation and integration." Proceedings of the National Academy of Sciences of the United States of America 104(33): 13507-13512.

Gaume, A., Vialatte, A., Mora-Sánchez, A., Ramdani, C., Vialatte, F.B., 2016. A psychoengineering paradigm for the neurocognitive mechanisms of biofeedback and neurofeedback. Neuroscience \& Biobehavioral Reviews 68, 891-910. https://doi.org/10.1016/j.neubiorev.2016.06.012

Gerin, M. I., H. Fichtenholtz, A. Roy, C. J. Walsh, J. H. Krystal, S. Southwick and M. Hampson (2016). "Real-Time fMRI Neurofeedback with War Veterans with Chronic PTSD: A Feasibility Study." Front Psychiatry 7: 111.

Gevensleben, H., B. Holl, B. Albrecht, D. Schlamp, O. Kratz, P. Studer, S. Wangler, A. Rothenberger, G. $\mathrm{H}$. Moll and H. Heinrich (2009). "Distinct EEG effects related to neurofeedback training in children with ADHD: A randomized controlled trial." International Journal of Psychophysiology 74(2): 149157.

Goldway, N., J. Ablin, O. Lubin, Y. Zamir, J. N. Keynan, A. Or-Borichev, M. Cavazza, F. Charles, N. Intrator, S. Brill, E. Ben-Simon, H. Sharon and T. Hendler (2019). "Volitional limbic neuromodulation exerts a beneficial clinical effect on Fibromyalgia." Neuroimage 186: 758-770.

Gross, J. J. and O. P. John (2003). "Individual differences in two emotion regulation processes: implications for affect, relationships, and well-being." J Pers Soc Psychol 85(2): 348-362.

Guan, M., L. Ma, L. Li, B. Yan, L. Zhao, L. Tong, S. Dou, L. Xia, M. Wang and D. Shi

(2015). "Self-regulation of brain activity in patients with postherpetic neuralgia: a double-blind randomized study using real-time fMRI neurofeedback." PLoS One 10. 
Halder, S., D. Agorastos, R. Veit, E. M. Hammer, S. Lee, B. Varkuti, M. Bogdan, W. Rosenstiel, N. Birbaumer and A. Kubler (2011). "Neural mechanisms of brain-computer interface control." Neuroimage 55(4): 1779-1790.

Hammer, E. M., S. Halder, B. Blankertz, C. Sannelli, T. Dickhaus, S. Kleih, K. R. Muller and A. Kubler (2012). "Psychological predictors of SMR-BCI performance." Biol Psychol 89(1): 80-86.

Hardman, E., J. Gruzelier, K. Cheesman, C. Jones, D. Liddiard, H. Schleichert and N. Birbaumer (1997). "Frontal interhemispheric asymmetry: self regulation and individual differences in humans." Neurosci Lett 221(2-3): 117-120.

Hellrung, L., A. Dietrich, M. Hollmann, B. Pleger, C. Kalberlah, E. Roggenhofer, A. Villringer and A. Horstmann (2018). "Intermittent compared to continuous real-time fMRI neurofeedback boosts control over amygdala activation." Neuroimage 166: 198-208. Herwig, U., J. Lutz, S. Scherpiet, H. Scheerer, J. Kohlberg, S. Opialla, A. Preuss, V. R. Steiger, J. Sulzer, S. Weidt, P. Stampfli, M. Rufer, E. Seifritz, L. Jancke and A. B. Bruhl

(2019). "Training emotion regulation through real-time fMRI neurofeedback of amygdala activity." Neuroimage 184: 687-696.

Ihssen, N., M. O. Sokunbi, A. D. Lawrence, N. S. Lawrence and D. E. J. Linden (2016).

"Neurofeedback of visual food cue reactivity: a potential avenue to alter incentive sensitization and craving." Brain imaging and behavior 11(3): 915-924.

Gross, J. J. and O. P. John (2003). "Individual differences in two emotion regulation processes: implications for affect, relationships, and well-being." J Pers Soc Psychol 85(2): 348-362.

Guan, M., L. Ma, L. Li, B. Yan, L. Zhao, L. Tong, S. Dou, L. Xia, M. Wang and D. Shi

(2015). "Self-regulation of brain activity in patients with postherpetic neuralgia: a double-blind randomized study using real-time fMRI neurofeedback." PLoS One 10.

Halder, S., D. Agorastos, R. Veit, E. M. Hammer, S. Lee, B. Varkuti, M. Bogdan, W. Rosenstiel, N. Birbaumer and A. Kubler (2011). "Neural mechanisms of brain-computer interface control." Neuroimage 55(4): 1779-1790.

Hammer, E. M., S. Halder, B. Blankertz, C. Sannelli, T. Dickhaus, S. Kleih, K. R. Muller and A. Kubler (2012). "Psychological predictors of SMR-BCl performance." Biol Psychol 89(1): 80-86.

Hardman, E., J. Gruzelier, K. Cheesman, C. Jones, D. Liddiard, H. Schleichert and N. Birbaumer (1997). "Frontal interhemispheric asymmetry: self regulation and individual differences in humans." Neurosci Lett 221(2-3): 117-120.

Hellrung, L., A. Dietrich, M. Hollmann, B. Pleger, C. Kalberlah, E. Roggenhofer, A. Villringer and A. Horstmann (2018). "Intermittent compared to continuous real-time fMRI neurofeedback boosts control over amygdala activation." Neuroimage 166: 198-208. Herwig, U., J. Lutz, S. Scherpiet, H. Scheerer, J. Kohlberg, S. Opialla, A. Preuss, V. R. Steiger, J. Sulzer, S. Weidt, P. Stampfli, M. Rufer, E. Seifritz, L. Jancke and A. B. Bruhl (2019). "Training emotion regulation through real-time fMRI neurofeedback of amygdala activity." Neuroimage 184: 687-696.

Ihssen, N., M. O. Sokunbi, A. D. Lawrence, N. S. Lawrence and D. E. J. Linden (2016). "Neurofeedback of visual food cue reactivity: a potential avenue to alter incentive sensitization and craving." Brain imaging and behavior 11(3): 915-924. 
Keynan, J. N., Y. Meir-Hasson, G. Gilam, A. Cohen, G. Jackont, S. Kinreich, L. Ikar, A. Or-Borichev, A. Etkin, A. Gyurak, I. Klovatch, N. Intrator and T. Hendler (2016). "Limbic Activity Modulation Guided by Functional Magnetic Resonance Imaging-Inspired Electroencephalography Improves Implicit Emotion Regulation." Biol Psychiatry 80(6): 490-496.

Kirsch, M., I. Gruber, M. Ruf, F. Kiefer and P. Kirsch (2016). "Real-time functional magnetic resonance imaging neurofeedback can reduce striatal cue-reactivity to alcohol stimuli." Addict Biol 21(4): 982992.

Kober, H., L. F. Barrett, J. Joseph, E. Bliss-Moreau, K. Lindquist and T. D. Wager (2008). "Functional grouping and cortical-subcortical interactions in emotion: a meta-analysis of neuroimaging studies." Neuroimage 42(2): 998-1031.

Kober, S. E., M. Witte, M. Ninaus, K. Koschutnig, D. Wiesen, G. Zaiser, C. Neuper and G. Wood (2017). "Ability to Gain Control Over One's Own Brain Activity and its Relation to Spiritual Practice: A Multimodal Imaging Study." Front Hum Neurosci 11: 271.

Kober, S. E., M. Witte, M. Ninaus, C. Neuper and G. Wood (2013). "Learning to modulate one's own brain activity: the effect of spontaneous mental strategies." Front Hum Neurosci 7: 695.

Koizumi, A., K. Amano, A. Cortese, K. Shibata, W. Yoshida, B. Seymour, M. Kawato and H. Lau (2016). "Fear reduction without fear through reinforcement of neural activity that bypasses conscious exposure." Nat Hum Behav 1.

Koush, Y., D. E. Meskaldji, S. Pichon, G. Rey, S. W. Rieger, D. E. Linden, D. Van De Ville, P. Vuilleumier and F. Scharnowski (2017). "Learning Control Over Emotion Networks Through Connectivity-Based Neurofeedback." Cereb Cortex 27(2): 1193-1202.

Koush, Y., M. J. Rosa, F. Robineau, K. Heinen, W. R. S, N. Weiskopf, P. Vuilleumier, D. Van De Ville and F. Scharnowski (2013). "Connectivity-based neurofeedback: dynamic causal modeling for real-time fMRI." Neuroimage 81: 422-430.

Krause, F., C. Benjamins, M. Luehrs, J. Eck, Q. Noirhomme, M. Rosenke, S. Brunheim, B. Sorger and R. Goebel (2017). "Real-time fMRI-based self-regulation of brain activation across different visual feedback presentations." Brain Computer Interfaces 4(1-2): 87-101. LaConte, S. M. (2011). "Decoding fMRI brain states in real-time." Neuroimage 56(2): 440-454.

LaConte, S. M., S. J. Peltier and X. P. Hu (2007). "Real-time fMRI using brain-state classification." Human Brain Mapping 28: 1033-1044.

Lacroix, J.M., 1986. Mechanisms of Biofeedback Control, in: Consciousness and Self-Regulation. Springer US, pp. 137-162.

Lawrence, E. J., L. Su, G. J. Barker, N. Medford, J. Dalton, S. C. Williams, N. Birbaumer, R. Veit, S. Ranganatha, J. Bodurka, M. Brammer, V. Giampietro and A. S. David (2014). "Self-regulation of the anterior insula: Reinforcement learning using real-time fMRI neurofeedback." Neuroimage 88: 113124.

Leeb, R., F. Lee, C. Keinrath, R. Scherer, H. Bischof and G. Pfurtscheller (2007). "Brain-computer communication: motivation, aim, and impact of exploring a virtual apartment." IEEE Trans Neural Syst Rehabil Eng 15(4): 473-482.

Li, X., K. J. Hartwell, J. Borckardt, J. J. Prisciandaro, M. E. Saladin, P. S. Morgan, K. A. Johnson, T. Lematty, K. T. Brady and M. S. George (2013). "Volitional reduction of anterior cingulate cortex 
activity produces decreased cue craving in smoking cessation: a preliminary real-time fMRI study." Addict Biol 18(4): 739-748.

Linden, D. E., I. Habes, S. J. Johnston, S. Linden, R. Tatineni, L. Subramanian, B. Sorger, D. Healy and R. Goebel (2012). "Real-time self-regulation of emotion networks in patients with depression." PLoS One 7(6): e38115.

Linhartova, P., A. Latalova, B. Kosa, T. Kasparek, C. Schmahl and C. Paret (2019). "fMRI neurofeedback in emotion regulation: A literature review." Neuroimage 193: 75-92. Lubianiker, N., N. Goldway, T. Fruchtman-Steinbok, C. Paret, J. N. Keynan, N. Singer, A. Cohen, K. Cohen Kadosh, D. E. J. Linden and T. Hendler (in press). "Process-based neurofeedback: towards precise endogenous neuromodulation." Nature Human Behavior. MacDuffie, K. E., J. Maclnnes, K. C. Dickerson, K. M. Eddington, T. J. Strauman and R. A. Adcock (2018). "Single session real-time fMRI neurofeedback has a lasting impact on cognitive behavioral therapy strategies." Neuroimage Clin 19: 868-875.

Marxen, M., M. J. Jacob, D. K. Muller, S. Posse, E. Ackley, L. Hellrung, P. Riedel, S. Bender, R. Epple and M. N. Smolka (2016). "Amygdala Regulation Following fMRI-Neurofeedback without Instructed Strategies." Front Hum Neurosci 10: 183.

Menon, V. (2011). "Large-scale brain networks and psychopathology: a unifying triple network model." Trends Cogn Sci 15(10): 483-506.

Nan, W., J. P. Rodrigues, J. Ma, X. Qu, F. Wan, P. I. Mak, P. U. Mak, M. I. Vai and A. Rosa (2012). "Individual alpha neurofeedback training effect on short term memory." Int J Psychophysiol 86(1): 83-87.

Nicholson, A. A., D. Rabellino, M. Densmore, P. A. Frewen, C. Paret, R. Kluetsch, C. Schmahl, J. Theberge, R. W. Neufeld, M. C. McKinnon, J. Reiss, R. Jetly and R. A. Lanius (2017). "The neurobiology of emotion regulation in posttraumatic stress disorder: Amygdala downregulation via real-time fMRI neurofeedback." Hum Brain Mapp 38(1): 541-560. Paret, C., Hendler, T., under review. Live from the "regulating brain": Harnessing the brain to change emotion. Emotion Special Issue: Emotion Regulation (invited contribution).

Paret, C., R. Kluetsch, M. Ruf, T. Demirakca, S. Hoesterey, G. Ende and C. Schmahl (2014). "Downregulation of amygdala activation with real-time fMRI neurofeedback in a healthy female sample." Frontiers in Behavioral Neuroscience 8.

Paret, C., R. Kluetsch, J. Zaehringer, M. Ruf, T. Demirakca, M. Bohus, G. Ende and C. Schmahl (2016). "Alterations of amygdala-prefrontal connectivity with real-time fMRI neurofeedback in BPD patients." Soc Cogn Affect Neurosci 11(6): 952-960.

Paret, C., M. Ruf, M. F. Gerchen, R. Kluetsch, T. Demirakca, M. Jungkunz, K. Bertsch, C. Schmahl and G. Ende (2016). "fMRI neurofeedback of amygdala response to aversive stimuli enhances prefrontallimbic brain connectivity." Neuroimage 125: 182-188.

Paret, C., J. Zahringer, M. Ruf, M. F. Gerchen, S. Mall, T. Hendler, C. Schmahl and G. Ende (2018). "Monitoring and control of amygdala neurofeedback involves distributed information processing in the human brain." Hum Brain Mapp.

Paret, C., Zähringer, J., Ruf, M., Ende, G., Schmahl, C., 2019. The Orbitofrontal Cortex Processes Neurofeedback Failure Signals. Behavioural Brain Research 111938.

https://doi.org/10.1016/j.bbr.2019.111938 
Powers, J. P. and K. S. LaBar (2019). "Regulating emotion through distancing: A taxonomy, neurocognitive model, and supporting meta-analysis." Neurosci Biobehav Rev 96: 155-173. Radua, J., T. Stoica, D. Scheinost, C. Pittenger and M. Hampson (2016). "Neural correlates of success and failure signals during neurofeedback learning." Neuroscience.

Ramot, M., S. Grossman, D. Friedman and R. Malach (2016). "Covert neurofeedback without awareness shapes cortical network spontaneous connectivity." Proc Natl Acad Sci U S A 113(17): E2413-2420.

Randell, E., R. McNamara, L. Subramanian, K. Hood and D. Linden (2018). "Current practices in clinical neurofeedback with functional MRI-Analysis of a survey using the TIDieR checklist." Eur Psychiatry 50: 28-33.

Raz, G., Y. Winetraub, Y. Jacob, S. Kinreich, A. Maron-Katz, G. Shaham, I. Podlipsky, G. Gilam, E. Soreq and T. Hendler (2012). "Portraying emotions at their unfolding: a multilayered approach for probing dynamics of neural networks." Neuroimage 60(2): 1448-1461.

Ros, T., S. Enriquez-Geppert, V. Zotev, K. Young, Wood, G., , S. Whitfield-Gabrieli, F. Wan, F. Vialatte, D. Van De Ville, D. Todder and ... (pre-print). "Consensus on the reporting and experimental design of clinical and cognitive-behavioural neurofeedback studies (CRED-nf checklist)." PsyArXiv.

Rota, G., G. Handjaras, R. Sitaram, N. Birbaumer and G. Dogil (2011). "Reorganization of functional and effective connectivity during real-time $\mathrm{fMRI}-\mathrm{BCl}$ modulation of prosody processing." Brain Lang 117(3): 123-132.

Rubia, K., A. B. Smith, M. J. Brammer and E. Taylor (2003). "Right inferior prefrontal cortex mediates response inhibition while mesial prefrontal cortex is responsible for error detection." Neuroimage 20(1): 351-358.

Sauer, C., G. Sheppes, H. K. Lackner, E. A. Arens, R. Tarrasch and S. Barnow (2016).

"Emotion regulation choice in female patients with borderline personality disorder: Findings from self-reports and experimental measures." Psychiatry Res 242: 375-384.

Schabus, M. (2017). "Reply: On assessing neurofeedback effects: should double-blind replace neurophysiological mechanisms?" Brain 140(10): e64.

Schabus, M., H. Griessenberger, M. T. Gnjezda, D. P. J. Heib, M. Wislowska and K. Hoedlmoser (2017). "Better than sham? A double-blind placebo-controlled neurofeedback study in primary insomnia." Brain 140(4): 1041-1052.

Scharnowski, F., C. Hutton, O. Josephs, N. Weiskopf and G. Rees (2012). "Improving visual perception through neurofeedback." J Neurosci 32(49): 17830-17841.

Scharnowski, F., R. Veit, R. Zopf, P. Studer, S. Bock, J. Diedrichsen, R. Goebel, K. Mathiak, N. Birbaumer and N. Weiskopf (2015). "Manipulating motor performance and memory through realtime fMRI neurofeedback." Biol Psychol 108: 85-97.

Scharnowski, F. and N. Weiskopf (2015). "Cognitive enhancement through real-time fMRI neurofeedback." Current Opinion in Behavioral Science.

Scheinost, D., T. Stoica, S. Wasylink, P. Gruner, J. Saksa, C. Pittenger and M. Hampson 
(2014). "Resting state functional connectivity predicts neurofeedback response." Front Behav Neurosci 8: 338.

Sepulveda, P., R. Sitaram, M. Rana, C. Montalba, C. Tejos and S. Ruiz (2016). "How feedback, motor imagery, and reward influence brain self-regulation using real-time fMRI." Hum Brain Mapp 37(9): 3153-3171.

Sheppes, G., S. Scheibe, G. Suri and J. J. Gross (2011). "Emotion-regulation choice." Psychol Sci 22(11): 1391-1396.

Sheppes, G., S. Scheibe, G. Suri, P. Radu, J. Blechert and J. J. Gross (2014). "Emotion regulation choice: a conceptual framework and supporting evidence." J Exp Psychol Gen 143(1): 163-181.

Sherlin, L.H., Arns, M., Lubar, J., Heinrich, H., Kerson, C., Strehl, U., Sterman, M.B., 2011. Neurofeedback and Basic Learning Theory: Implications for Research and Practice. Journal of Neurotherapy 15, 292-304. https://doi.org/10.1080/10874208.2011.623089

Shibata, K., G. Lisi, A. Cortese, T. Watanabe, Y. Sasaki and M. Kawato (2019). "Toward a comprehensive understanding of the neural mechanisms of decoded neurofeedback." Neuroimage 188: 539-556.

Shibata, K., T. Watanabe, M. Kawato and Y. Sasaki (2016). "Differential Activation Patterns in the Same Brain Region Led to Opposite Emotional States." PLoS Biol 14(9): e1002546. Shibata, K., T. Watanabe, Y. Sasaki and M. Kawato (2011). "Perceptual learning incepted by decoded fMRI neurofeedback without stimulus presentation." Science 334(6061): 1413-1415.

Sitaram, R., T. Ros, L. Stoeckel, S. Haller, F. Scharnowski, J. Lewis-Peacock, N. Weiskopf, M. L. Blefari, M. Rana, E. Oblak, N. Birbaumer and J. Sulzer (2017). "Closed-loop brain training: the science of neurofeedback." Nat Rev Neurosci 18(2): 86-100.

Sitaram, R., R. Veit, B. Stevens, A. Caria, C. Gerloff, N. Birbaumer and F. Hummel (2012). "Acquired control of ventral premotor cortex activity by feedback training: an exploratory real-time FMRI and TMS study." Neurorehabil Neural Repair 26(3): 256-265.

Skottnik, L., B. Sorger, T. Kamp, D. Linden and R. Goebel (2019). "Success and failure of controlling the real-time functional magnetic resonance imaging neurofeedback signal are reflected in the striatum." Brain Behav 9(3): e01240.

Sorger, B., J. Reithler, B. Dahmen and R. Goebel (2012). "A real-time fMRI-based spelling device immediately enabling robust motor-independent communication." Curr Biol 22(14): 1333-1338.

Sorger, B., F. Scharnowski, D. E. J. Linden, M. Hampson and K. D. Young (2019). "Control freaks: Towards optimal selection of control conditions for fMRI neurofeedback studies." Neuroimage 186: 256-265.

Spetter, M. S., R. Malekshahi, N. Birbaumer, M. Luhrs, A. H. van der Veer, K. Scheffler, S. Spuckti, H. Preissl, R. Veit and M. Hallschmid (2017). "Volitional regulation of brain responses to food stimuli in overweight and obese subjects: A real-time fMRI feedback study." Appetite 112: 188-195.

Staunton, G. and K. Cohen Kadosh (2019). "Which psychological factors influence learning outcomes from neurofeedback- a literature review." Neuroimage 15: 545-555.

Strehl, U., 2014. What learning theories can teach us in designing neurofeedback treatments. Front Hum Neurosci 8, 894. https://doi.org/10.3389/fnhum.2014.00894 
Subramanian, L., J. Hindle, S. Johnston, M. Roberts, M. Husain, R. Goebel and D. E. J. Linden (2011). "Real-time fMRI Neurofeedback for Treatment of Parkinson's Disease." Journal of Neuroscience 31(45): 16309-16317.

Sulzer, J., S. Haller, F. Scharnowski, N. Weiskopf, N. Birbaumer, M. L. Blefari, A. B. Bruehl, L. G. Cohen, R. C. DeCharms, R. Gassert, R. Goebel, U. Herwig, S. LaConte, D. Linden, A. Luft, E. Seifritz and R. Sitaram (2013). "Real-time fMRI neurofeedback: progress and challenges." Neuroimage 76: 386399.

Thibault, R. T., M. Lifshitz and A. Raz (2016). "The self-regulating brain and neurofeedback: Experimental science and clinical promise." Cortex 74: 247-261.

Thibault, R. T., M. Lifshitz and A. Raz (2018). "The climate of neurofeedback: scientific rigour and the perils of ideology." Brain 141(2): e11.

Thibault, R. T. and A. Raz (2016). "When can neurofeedback join the clinical armamentarium?" The Lancet. Psychiatry 3(6): 497-498.

Thomas, L. A., M. D. De Bellis, R. Graham and K. S. LaBar (2007). "Development of emotional facial recognition in late childhood and adolescence." Developmental Science 10(5): 547-558.

Van den Boom, M. A., J. M. Jansma and N. F. Ramsey (2018). "Rapid acquisition of dynamic control over DLPFC using real-time fMRI feedback." European Neuropsychopharmacology.

Veit, R., V. Singh, R. Sitaram, A. Caria, K. Rauss and N. Birbaumer (2012). "Using real-time fMRI to learn voluntary regulation of the anterior insula in the presence of threat-related stimuli." Soc Cogn Affect Neurosci 7(6): 623-634.

Watanabe, T., Y. Sasaki, K. Shibata and M. Kawato (2017). "Advances in fMRI Real-Time Neurofeedback." Trends Cogn Sci 21(12): 997-1010.

Witte, M., S. E. Kober, M. Ninaus, C. Neuper and G. Wood (2013). "Control beliefs can predict the ability to up-regulate sensorimotor rhythm during neurofeedback training." Front Hum Neurosci 7: 478.

Yamin, H. G., T. Gazit, N. Tchemodanov, G. Raz, G. Jackont, F. Charles, I. Fried, T. Hendler and M. Cavazza (2017). "Depth electrode neurofeedback with a virtual reality interface." Brain-Computer Interfaces 4(4): 201-213.

Yin, H. H., S. P. Mulcare, M. R. Hilario, E. Clouse, T. Holloway, M. I. Davis, A. C. Hansson, D. M. Lovinger and R. M. Costa (2009). "Dynamic reorganization of striatal circuits during the acquisition and consolidation of a skill." Nat Neurosci 12(3): 333-341. Young, K. D., V. Zotev, R. Phillips, M. Misaki, H. Yuan, W. C. Drevets and J. Bodurka

(2014). "Real-time FMRI neurofeedback training of amygdala activity in patients with major depressive disorder." PLoS One 9(2): e88785.

Yuan, H., K. D. Young, R. Phillips, V. Zotev, M. Misaki and J. Bodurka (2014). "Resting-state functional connectivity modulation and sustained changes after real-time functional magnetic resonance imaging neurofeedback training in depression." Brain Connect 4(9): 690-701.

Zhang, G., L. Yao, H. Zhang, Z. Long and X. Zhao (2013). "Improved working memory performance through self-regulation of dorsal lateral prefrontal cortex activation using real-time fMRI." PLoS One 8(8): e73735. 
Zilverstand, A., B. Sorger, P. Sarkheil and R. Goebel (2015). "fMRI neurofeedback facilitates anxiety regulation in females with spider phobia." Front Behav Neurosci 9: 148.

Zotev, V., F. Krueger, R. Phillips, R. P. Alvarez, W. K. Simmons, P. Bellgowan, W. C. Drevets and J. Bodurka (2011). "Self-regulation of amygdala activation using real-time FMRI neurofeedback." PLoS One 6(9): e24522.

Zotev, V., R. Phillips, K. D. Young, W. C. Drevets and J. Bodurka (2013). "Prefrontal control of the amygdala during real-time fMRI neurofeedback training of emotion regulation." PLoS One 8(11): e79184.

\section{Figures}

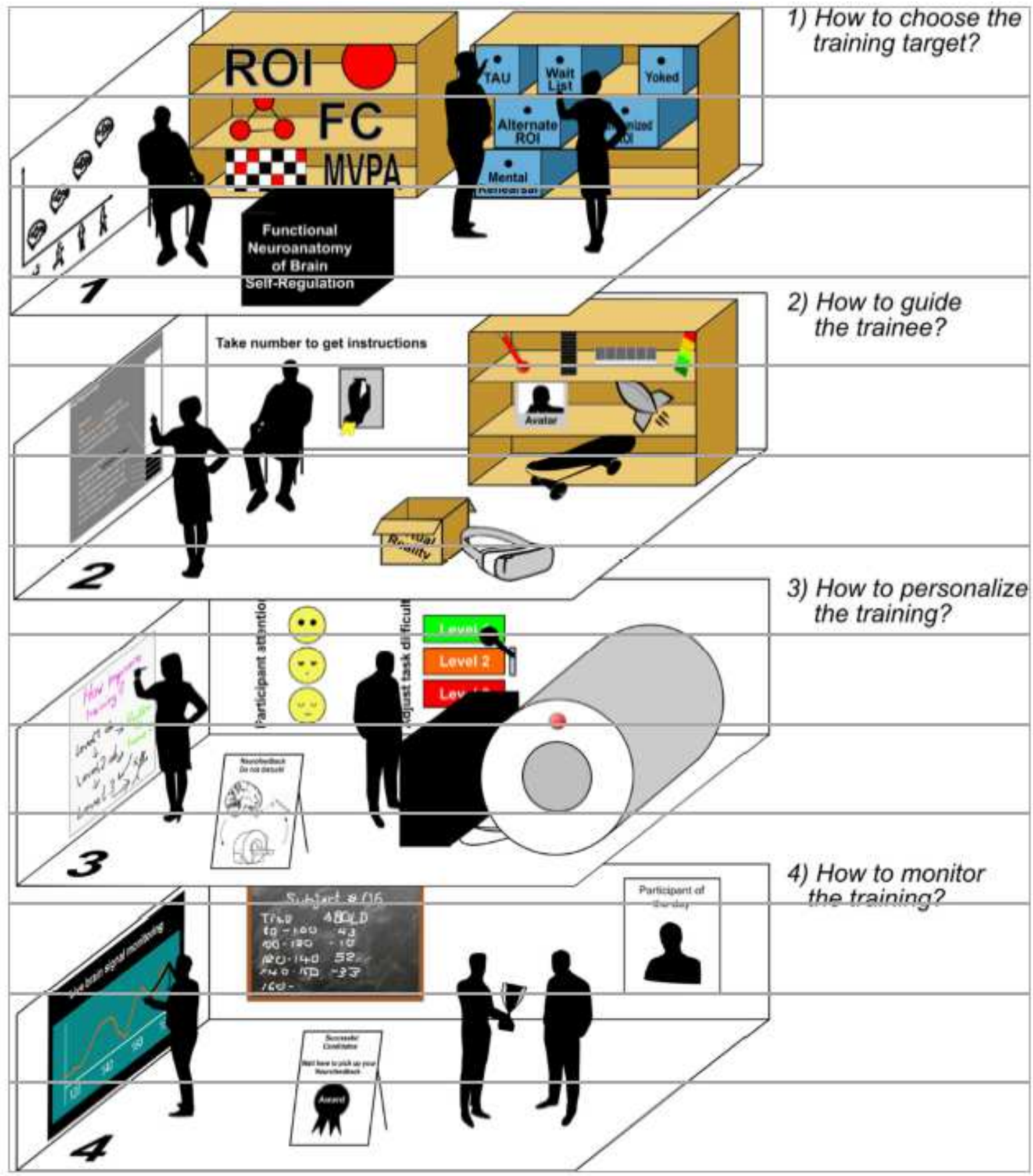


Figure 1. "Floor plan", illustrating domains of neurofeedback procedures of current debate and discussed in this article.

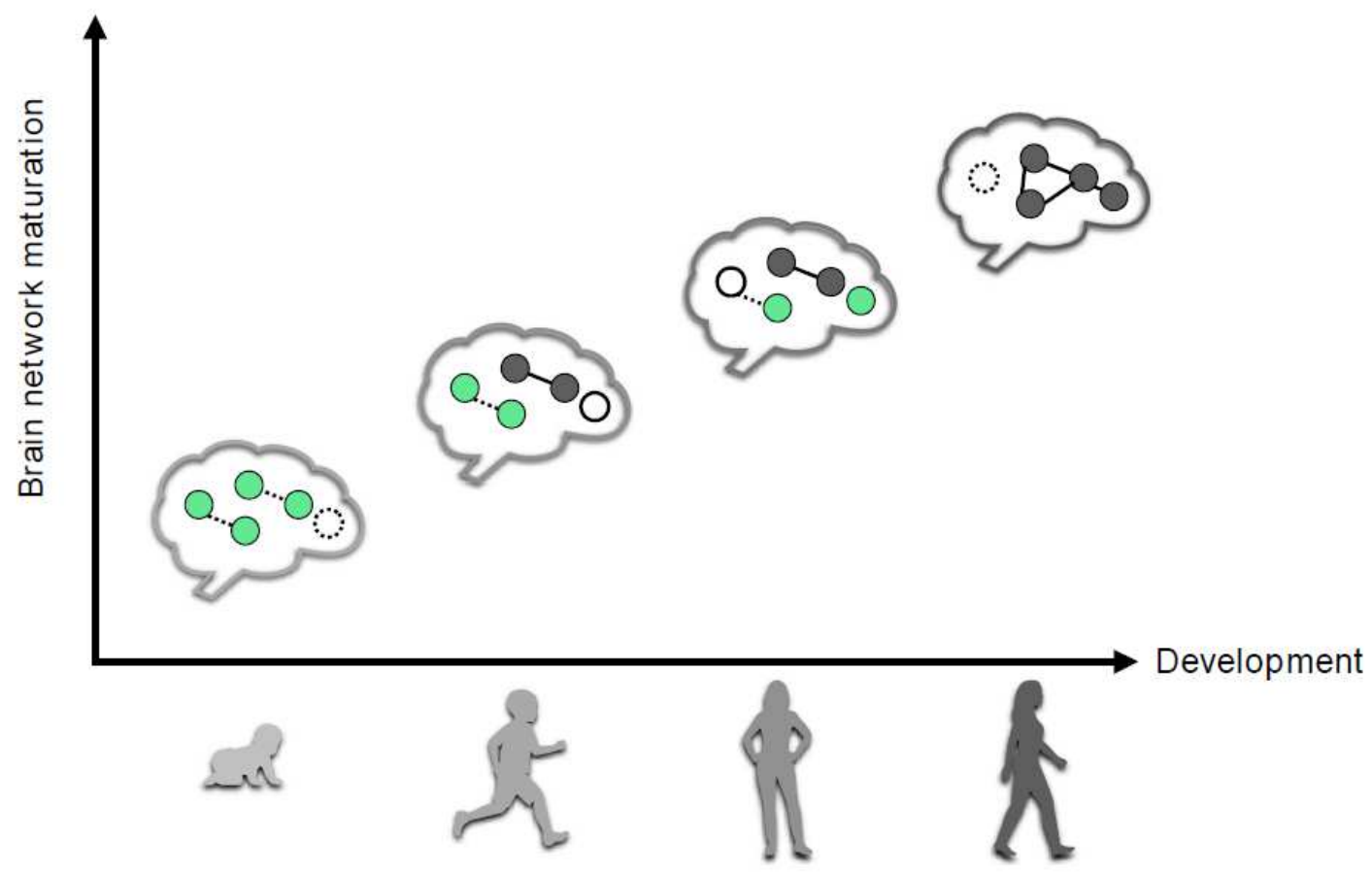

Figure 2. How to treat the maturing brain: train networks specific for a particular developmental stage or reinforce adult healthy brain function? Brain networks undergo considerable maturation throughout the first two decades of life (Fair, Cohen et al. 2009, Cohen Kadosh, Cohen Kadosh et al. 2011), a change which is reflected in the functional and structural connectivity patterns across the age range. For the design of effective NF protocols, this could mean that different brain regions may support a specific cognitive function at each age point. The question therefore arises whether one should teach young individuals to regulate brain responses that would support a cognitive function at the specific developmental stage, or, instead teach them directly to use the brain network that supports this ability in healthy mature adults. This may seem especially important if one considers the developing brain as an adaptive system, where the networks of brain regions that support cognitive abilities change interactively as a result of on-going brain maturation and cognitive development (Cohen Kadosh and Johnson 2007, Johnson, Grossmann et al. 2009). The same principle applies for clinical populations, which may rely on alternative brain networks to perform a task. Hence, the (temporary) use of an alternative brain network can be considered both logical and adaptive and it remains to be determined whether NF should target these (current) alternatives instead of typical key regions (see also discussion in Lubianker et al., in press). 


\section{A)}
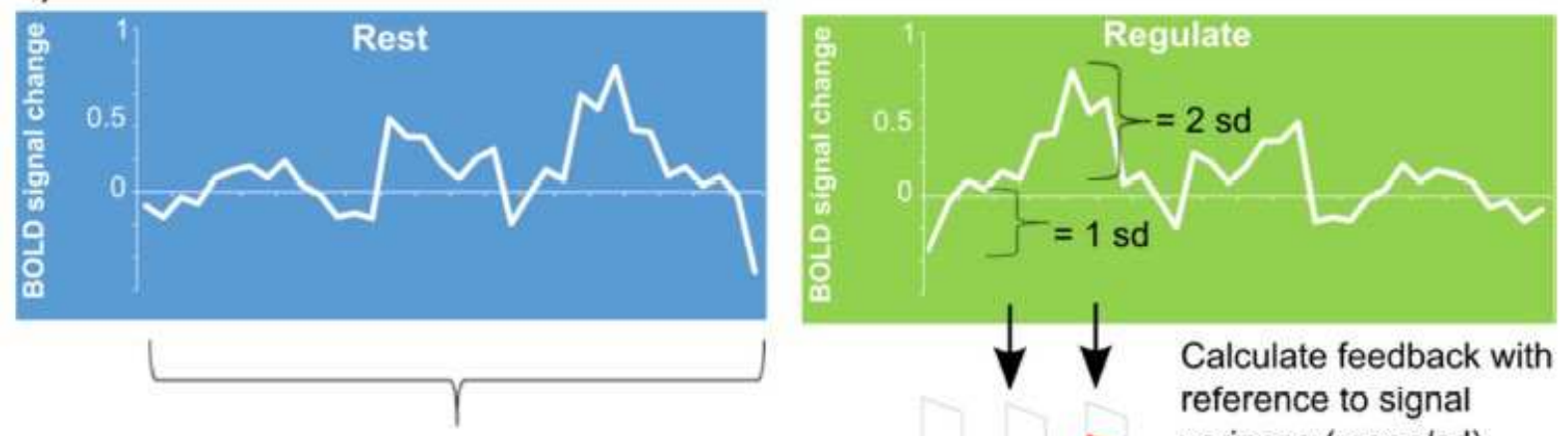

Calculate mean and sd of signal reference to signal variance (mean/sd) during 'rest'

\section{B)}
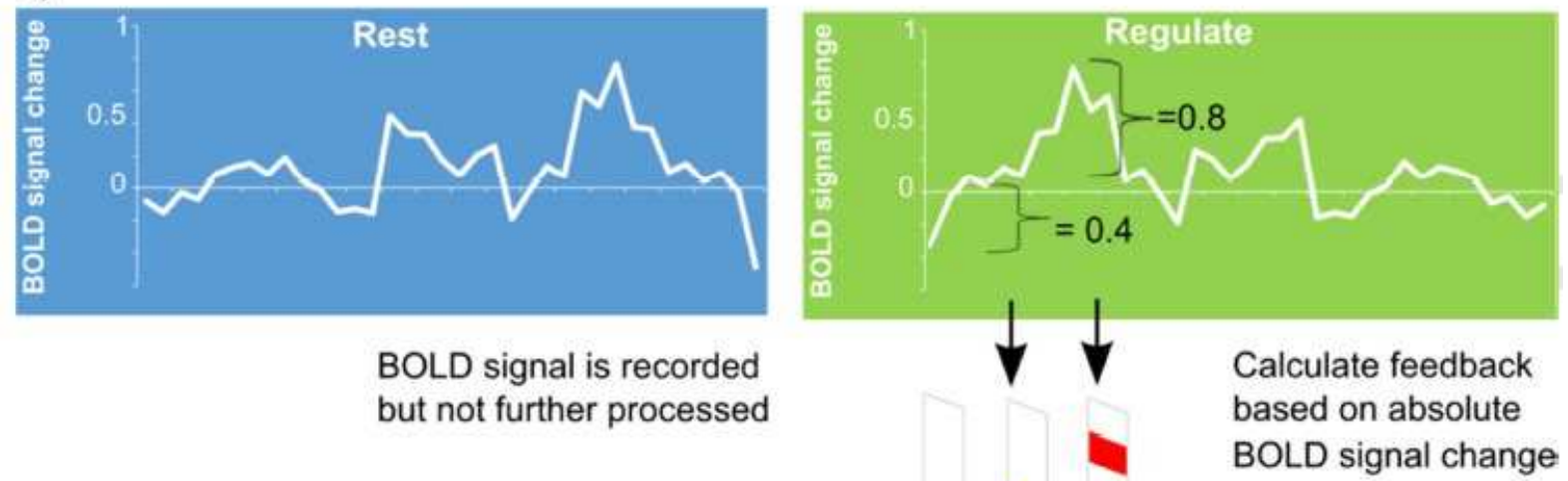

Figure 3. Feedback is calculated based on the subject's BOLD signal variance or based on absolute signal change. A) The BOLD signal is sampled during a period (e.g. during 'rest', left area in blue). Mean and standard deviation (sd) is calculated and used to standardize feedback during the 'regulate' trial (right area in green). B) An absolute threshold is set to decide, whether BOLD signal change is followed by a change in the feedback thermometer.

A)
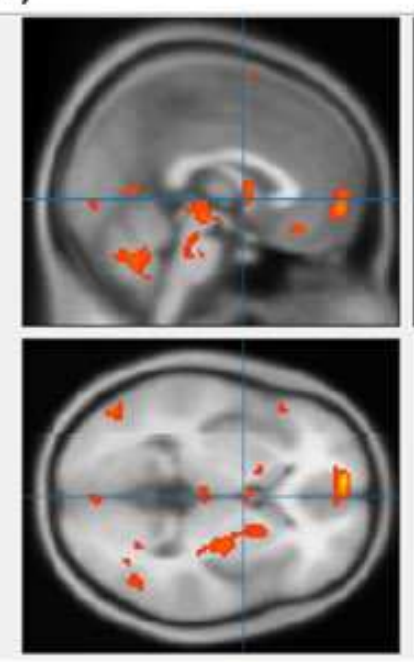

B)
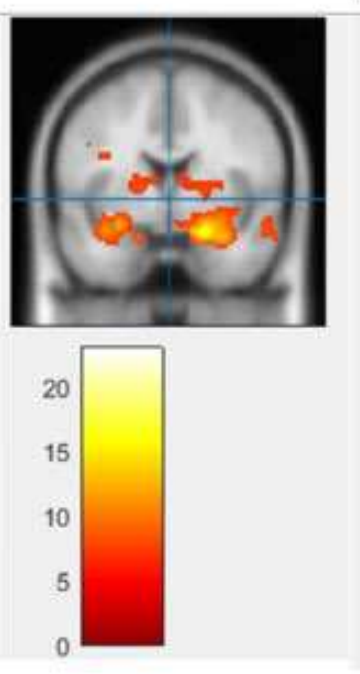
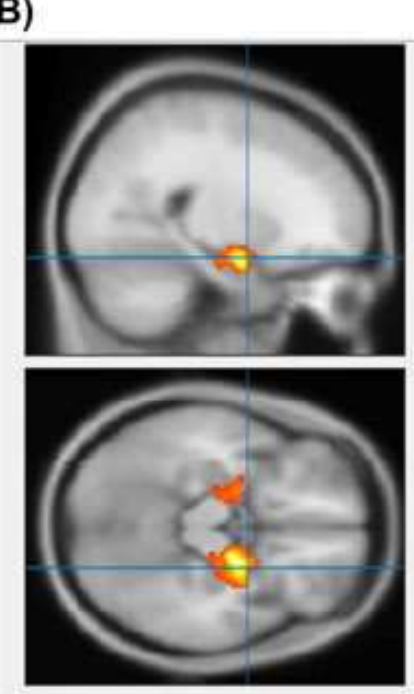
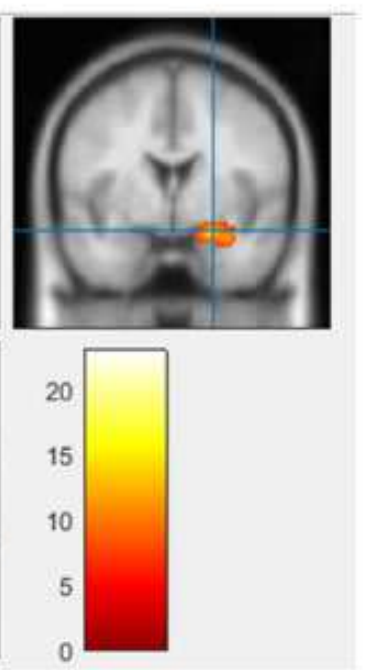
Figure 4. Different real-time signal processing pipelines yield feedback that is correlated with different regional activation patterns. For illustration, right amygdala activation was calculated with two different real-time preprocessing routines, based on prerecorded fMRI data of $\mathrm{N}=17$ subjects who were administered an fMRI emotion regulation experiment (Linhartová, Gerchen \& Paret, unpublished data). The resulting amygdala-signal was used as a predictor in a whole-brain statistical parametric mapping analysis. The maps show results from the group t-test ( $\mathrm{P}<0.05 \mathrm{FWE}$-corrected; colour-scale: $\mathrm{t}$-values). The map shows regions that are significantly correlated with the amygdala signal as it was received by real-time analyses. Hence, activation of these brain regions is represented in this signal, and would have been reflected in feedback from this signal (note that subjects in this study did not receive feedback). A) The amygdala signal was cleaned by real-time regression of movement parameters and "censoring" of super-threshold movements, based on realignment regressors. B) Activation from a rectangular control region was subtracted from the amygdala signal (see Paret et al. 2018 for comprehensive description of methods).

A) Fixed threshold

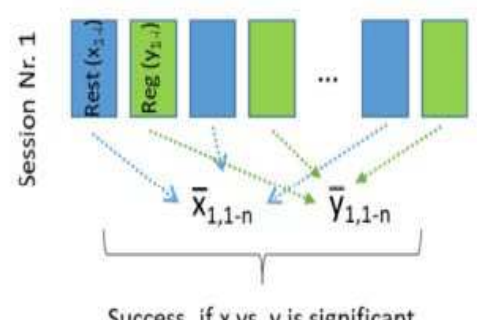

Success, if $x$ vs. $y$ is significant
B) Success rate

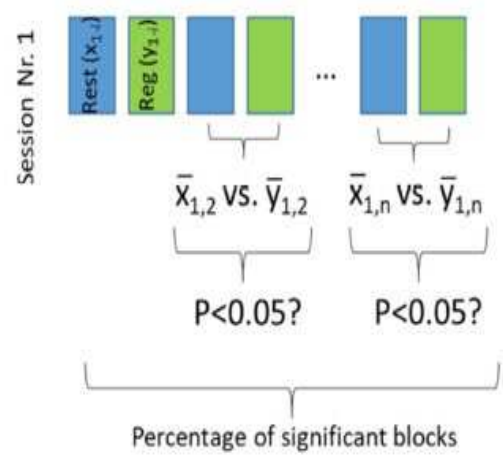

C) Personal effect size

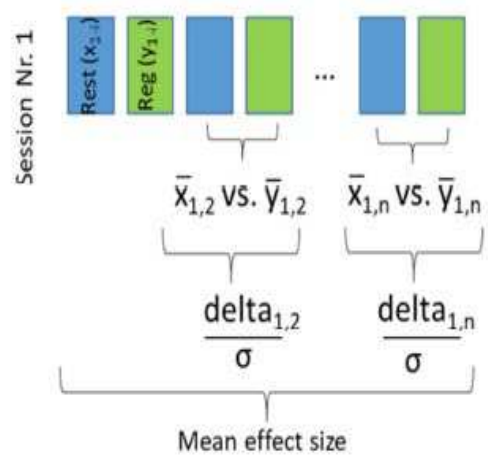

Figure 5. illustrates different approaches to calculate success metrics. An experiment of alternating 'rest' (blue) and 'regulate' (reg; green) trials is shown. A 'rest' and subsequent 'reg' trial form together a block-unit. The signal of each trial is congregated for 'rest' (i.e., samples $1,2, \ldots, \mathrm{i} ; \mathrm{x} 1-\mathrm{i}$ ) and ,reg' (i.e., samples $1,2, \ldots, \mathrm{i}$; $\mathrm{y}_{1-\mathrm{i}}$ ) to result in a mean value (e.g. $\mathrm{x}\left(\right.$ stroked $_{1, \mathrm{n}}$ is the mean of all ,rest ${ }^{6}$-samples in session 1, block $\mathrm{n}$; $\mathrm{y}$ (stroked) $1, \mathrm{n}$ is the mean of all ,reg'-samples in session 1, block n). A) For the ,fixed threshold' index, the mean of all samples in 'rest' $\left(\mathrm{x}\left(\right.\right.$ stroked $\left._{1, \mathrm{n}}\right)$ and ' $\mathrm{reg}^{\text {' }}\left(\mathrm{y}\left(\right.\right.$ stroked $\left._{1, \mathrm{n}}\right)$ is calculated and passed to a statistical test. If significant, regulation was successful. B) For the ,success rate', 'reg' and 'rest' conditions are compared in a block-wise manner. One receives a statistic for each block showing whether samples in the ,reg' trial differed significantly from the preceding, rest $^{\text {‘ }}$ trial. The success rate of a session is reflected by the percentage of blocks with signficant difference. C) For calculation of 'personal effect size", we take the difference (delta) between the , reg' and ,rest' mean of each block. To account for differences in signal-variance, one may divide each delta by the pooled standard deviation $\sigma$ of this block, and receive blockwise effect size estimates. The mean of these is the personal effect size of the session. 
Table 1. Overview on combinations of brain target and outcome analysis with single region and network NF trainings.

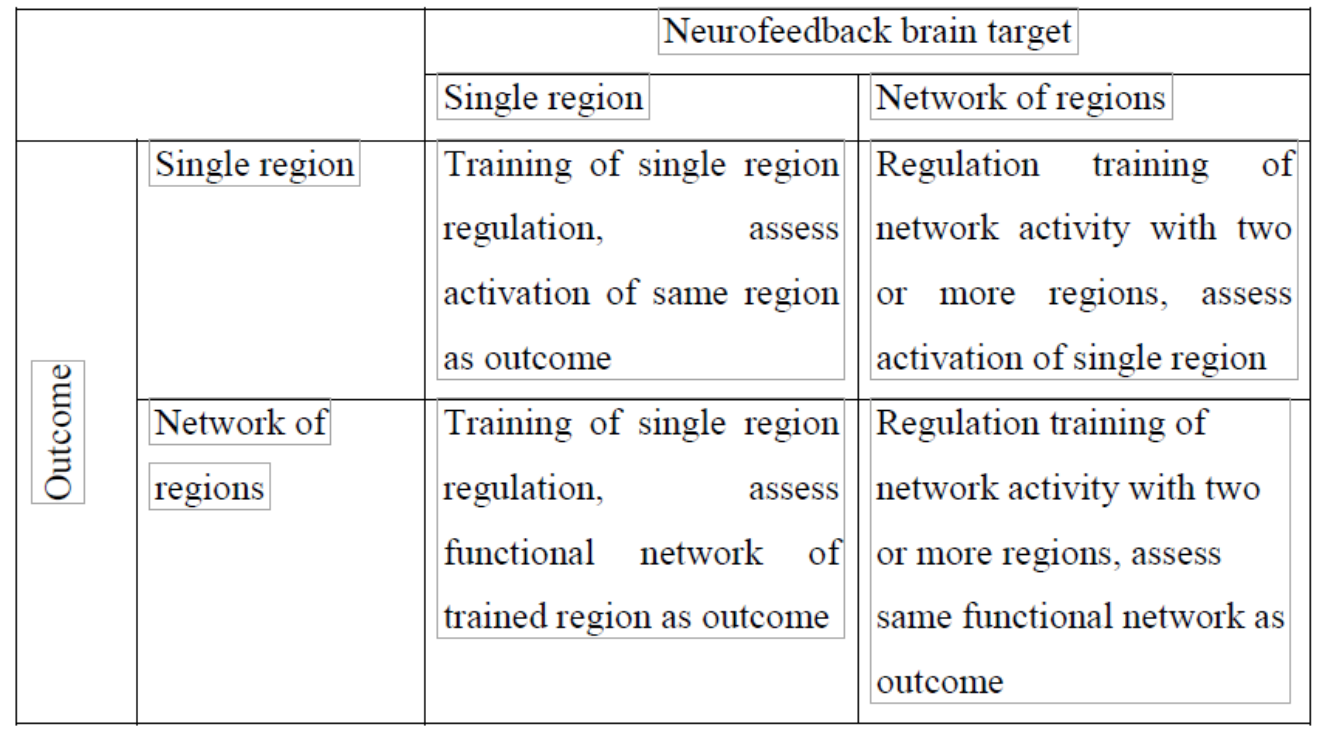

Table 2. Pros and cons of simple and more complex NF interfaces

\begin{tabular}{|c|c|c|}
\hline & & Virtual reality environment \\
\hline Examples & $\begin{array}{l}\text { Thermometer, } \\
\text { sound/music jingle, rocket } \\
\text { space ship, virtual fire }\end{array}$ & $\begin{array}{l}\text { Interact with avatar, reach a coffee } \\
\text { mug, navigate in computer game }\end{array}$ \\
\hline Pros & $\begin{array}{l}\text { Distractors are reduced to } \\
\text { minimum }\end{array}$ & $\begin{array}{l}\text { Improved learning } \\
\text { Better sustainability } \\
\text { Better transferability } \\
\text { Increased motivation }\end{array}$ \\
\hline Cons & May produce fatigue & $\begin{array}{l}\text { May distract from task } \\
\text { More complex scenarios come } \\
\text { with higher cognitive demands and } \\
\text { may turn out difficult for some } \\
\text { populations }\end{array}$ \\
\hline
\end{tabular}

Table 3. NF training success indices

\begin{tabular}{|l|l|l|}
\hline Index Type & Pros & Cons \\
\hline Single session & \\
\hline
\end{tabular}




\begin{tabular}{|c|c|c|}
\hline $\begin{array}{l}\text { Fixed Threshold; } \\
\text { T-test comparing between average } \\
\text { 'rest' and 'regulate' }\end{array}$ & $\begin{array}{l}\text { Provides definitive yes/no } \\
\text { answer }\end{array}$ & $\begin{array}{l}\text { Does not reflect higher/lower } \\
\text { levels of success } \\
\text { Threshold is often arbitrary; } \\
\text { might cause mislabeling in } \\
\text { ambiguous situations } \\
\text { Cannot be used to test } \\
\text { correlations with outcome } \\
\text { measures }\end{array}$ \\
\hline $\begin{array}{l}\text { Success rate; } \\
\text { Based on comparison of 'regulate' } \\
\text { to the previous 'rest' condition } \\
\text { using a t-test. The final result is } \\
\text { the sum/ratio of significant } \\
\text { 'regulate'-'rest' blocks. }\end{array}$ & $\begin{array}{l}\text { Assesses the consistency } \\
\text { of regulation } \\
\text { Less arbitrary, avoids } \\
\text { mislabeling in ambiguous } \\
\text { situations }\end{array}$ & $\begin{array}{l}\text { Ordinal: cannot be used for } \\
\text { parametric tests }\end{array}$ \\
\hline $\begin{array}{l}\text { Personal effect size; } \\
\text { Based on difference between } \\
\text { means of regulate and rest } \\
\text { conditions, divided by pooled } \\
\text { standard deviation. The result is } \\
\text { the mean of these effect sizes. }\end{array}$ & $\begin{array}{l}\text { Continuous } \\
\text { Noise "insensitive" }\end{array}$ & $\begin{array}{l}\text { Instable; } \\
\text { Sensitive to block duration and } \\
\text { sampling rate }\end{array}$ \\
\hline \multicolumn{3}{|l|}{ Multiple sessions } \\
\hline $\begin{array}{l}\text { Mean Performance; } \\
\text { The average score of the selected } \\
\text { success index across all sessions }\end{array}$ & $\begin{array}{l}\text { "global score" of brain } \\
\text { regulation ability } \\
\text { Contains information } \\
\text { regarding all the sessions }\end{array}$ & Insensitive to temporal dynamics \\
\hline $\begin{array}{l}\text { Last-first session; } \\
\text { The delta between the first and } \\
\text { last session }\end{array}$ & $\begin{array}{l}\text { Quantifies over all } \\
\text { learning progress }\end{array}$ & $\begin{array}{l}\text { Assumes that the last session is } \\
\text { necessarily the best one. } \\
\text { Ignores additional information }\end{array}$ \\
\hline
\end{tabular}




\begin{tabular}{|c|c|c|}
\hline & & from the rest of the sessions \\
\hline $\begin{array}{l}\text { Learning Slope; } \\
\text { Linear regression/repeated } \\
\text { measures ANOVA with number } \\
\text { of session as independent/within- } \\
\text { subject factor }\end{array}$ & $\begin{array}{l}\text { Accounts for temporal } \\
\text { dynamics } \\
\text { Contains information } \\
\text { regarding all the sessions }\end{array}$ & $\begin{array}{l}\text { Assumes linear improvement in } \\
\text { NF performance, that is not } \\
\text { supported by all models of } \\
\text { neurofeedback learning (see } \\
\text { Sitaram et al., 2017) }\end{array}$ \\
\hline $\begin{array}{l}\text { Best Performance; } \\
\text { The best session/block throughout } \\
\text { the NF training }\end{array}$ & $\begin{array}{l}\text { Reflects maximum } \\
\text { individual potential }\end{array}$ & Noise sensitive \\
\hline
\end{tabular}

Table 4. Selective summary of protocol aspects that may increase efficiency of NF training.

\begin{tabular}{|c|c|c|}
\hline Measure & Current state-of-the-art & Suggestions \\
\hline $\begin{array}{l}\text { Limit training to number of } \\
\text { sessions that are needed for } \\
\text { learning. }\end{array}$ & $\begin{array}{l}\text { Empirical data on optimal } \\
\text { number of session is } \\
\text { lacking. Studies are } \\
\text { designed based on similar } \\
\text { studies already published } \\
\text { and expert suggestions from } \\
\text { the literature. }\end{array}$ & $\begin{array}{l}\text { Future studies should report } \\
\text { between-session success } \\
\text { and/or learning curve. } \\
\text { Studies are needed to } \\
\text { identify learning curves and } \\
\text { define threshold-criteria. }\end{array}$ \\
\hline $\begin{array}{l}\text { Improve learning with } \\
\text { additional out-of-scanner } \\
\text { treatment }\end{array}$ & $\begin{array}{l}\text { Initial studies show } \\
\text { feasibility and benefits in } \\
\text { clinical samples. }\end{array}$ & $\begin{array}{l}\text { Conduct more studies to } \\
\text { test benefits and feasibility } \\
\text { of out-of-scanner practice. } \\
\text { Identify maximum number } \\
\text { of NF sessions needed in } \\
\text { such protocols. }\end{array}$ \\
\hline $\begin{array}{l}\text { Adapt feedback range to the } \\
\text { subject's performance }\end{array}$ & $\begin{array}{l}\text { Some studies use an } \\
\text { adaptive range and find } \\
\text { successful learning. Studies } \\
\text { to assess benefits of } \\
\text { adaptive versus fixed } \\
\text { feedback range and baseline }\end{array}$ & $\begin{array}{l}\text { Investigate effect of } \\
\text { adaptive feedback on brain } \\
\text { self-regulation and assess } \\
\text { potential additivie benefits } \\
\text { for transfer of learning. }\end{array}$ \\
\hline
\end{tabular}




\begin{tabular}{|l|l|l|}
\hline & levels are missing. & \\
\hline $\begin{array}{l}\text { Introduce performance-level } \\
\text { criterion (e.g. A: regulation } \\
\text { without challenge; B: regulation } \\
\text { with mild stressor; C: regulation }\end{array}$ & Not yet tested. & $\begin{array}{l}\text { Studies should assess } \\
\text { potential improvements } \\
\text { with strong stressor). Use }\end{array}$ \\
$\begin{array}{l}\text { performance-levels for } \\
\text { decisions such as passing to } \\
\text { next level of difficulty, or to } \\
\text { stop training. }\end{array}$ & & \\
\hline
\end{tabular}

